\title{
Lift-up, Kelvin-Helmholtz and Orr mechanisms in turbulent jets
}

\author{
Ethan Pickering ${ }^{1}$, Georgios Rigas ${ }^{1}$, Petrônio A. S. Nogueira ${ }^{2}$, André \\ V. G. Cavalieri ${ }^{2}$, Oliver T. Schmidt ${ }^{3}$, Tim Colonius ${ }^{1}$ \\ ${ }^{1}$ Mechanical Engineering, California Institute of Technology, Pasadena, CA USA \\ ${ }^{3}$ Mechanical Engineering, University of California, San Diego, La Jolla, CA USA \\ ${ }^{2}$ Instituto Tecnológico de Aeronáutica, São José dos Campos, Brazil
}

(Received $\mathrm{xx}$; revised $\mathrm{xx}$; accepted $\mathrm{xx}$ )

\begin{abstract}
Three amplification mechanisms present in turbulent jets, namely lift-up, KelvinHelmholtz, and Orr, are characterized via global resolvent analysis and spectral proper orthogonal decomposition (SPOD) over a range of Mach numbers. The lift-up mechanism in turbulent jets, recently analyzed by Nogueira et al. (2019) via local analysis, is dominant at low Strouhal number $(S t)$ and non-zero azimuthal wavenumbers $(m)$. In these limits, a global SPOD analysis reveals streamwise vortices and streaks similar to those found in turbulent wall-bounded flows. These structures are in qualitative agreement with the global resolvent analysis, which shows that they are a response to upstream forcing by streamwise vorticity near the nozzle exit. Analysis of mode shapes, energy content, and sensitivity analysis distinguish the three mechanisms and the regions of wavenumber-frequency space where each dominates. Finally, SPOD analysis of localized regions shows that the lift-up mechanism is present throughout the jet, with a dominant azimuthal wavenumber inversely proportional to streamwise distance from the nozzle, with streaks of azimuthal wavenumber exceeding five near the nozzle, and wavenumbers one and two most energetic far downstream of the potential core.
\end{abstract}

\section{Introduction}

Coherent structures in turbulence are largely responsible for the transport of mass, momentum, and energy, and the radiation of acoustic waves. Early observations of orderly structure were found by Mollo-Christensen (1967) and Crow \& Champagne (1971) in turbulent jets and Brown \& Roshko (1974) in planar mixing layers. Almost 50 years later, a full understanding of underlying mechanisms driving the generation and sustenance of coherent structures remain elusive, yet their connection to longstanding engineering problems such as jet acoustics (Jordan \& Colonius 2013) and drag in wall-bounded flows (Jiménez 2018) has become increasingly apparent.

Coherent structures in turbulence have generally been interpreted and modeled in terms of instabilities of (typically steady) basic flows. In jet turbulence, Crighton \& Gaster (1976) hypothesized that coherent structures could be described as linear instability modes of the mean flow via a modal analysis. Since, significant progress has been made computing modal and non-modal mechanisms with varying degrees of generality. Earlier work focused on parabolized stability equations (PSE), and Gudmundsson \& Colonius (2011) showed that PSE solutions for an experimentally measured jet mean flow yielded good predictions for the dominant frequency/azimuthal mode structures educed from a near-field caged microphone array. However, fully global studies, Garnaud et al. (2013) yield a stable spectrum (for jets that are not too highly heated), and instability mecha- 
nisms need to be characterized by transient (non-modal) growth. Resolvent analysis, by contrast, characterizes linear amplification of disturbances in the frequency domain, and is therefore easier to relate experimental mechanisms in both transitional and turbulent flows. McKeon \& Sharma (2010) proposed a resolvent framework for the turbulent case, where nonlinear interactions are regarded as forcing terms to a linearized operator that amplifies them according to the turbulent mean flow. Resolvent analysis of jets have by now been computed for a variety of jet mean flows (Jeun et al. 2016; Semeraro et al. 2016; Schmidt et al. 2018; Lesshafft et al. 2019).

These studies have shown the presence of two essentially different linear amplification mechanisms, one which can be associated with the traditional (parallel-flow) KelvinHelmholtz $(\mathrm{KH})$ instability and an Orr-type mechanism, also identified through PSE by Tissot et al. (2017). Schmidt et al. (2018) showed that the former KH mechanism could be represented at low rank, in the sense that there is a large separation between the dominant and subdominant gains, suggesting an intrinsic mechanism reminiscent of a modal instability. On the contrary, the Orr mechanism was not low rank, in that it is more representative of a non-modal mechanism. They also found a close correspondence between the resolvent spectrum and the spectral proper orthogonal decomposition (SPOD) modes educed from high-fidelity, large-eddy simulation (LES) data.

However, previous global resolvent computations neglected the lowest frequency region of the spectrum, owing largely to computational difficulties, i.e. the large spatial domains required. Recently, Nogueira et al. (2019) presented evidence, both through SPOD of particle image velocimetry data and a parallel resolvent analysis, that a third and uniquely distinct mechanism is at play in the low frequency region of the spectrum, namely the lift-up mechanism.

The lift-up mechanism and the associated coherent structures, streaks, have long been understood as an important mechanism in wall bounded flows (see review by Brandt (2014) and references therein). Parallel to the history of mechanism identification in jet flows, the lift-up mechanism (as well as Tollmien-Schlichting and Orr from other references) has been systematically described, first through inspirational observations (Klebanoff 1971; Kim et al. 1971), then local analyses (Moffatt 1965; Ellingsen \& Palm 1975; Landahl 1980), followed by transient growth (Butler \& Farrell 1992; Farrell \& Ioannou 1993), and most recently resolvent analysis (Hwang \& Cossu 2010; Abreu et al. 2019). The salient properties of the lift-up mechanism include streamwise vortices (rolls with streamwise vorticity $\omega_{x}^{\prime}$ ) that lift-up low speed fluid from the wall (and push high speed fluid toward the wall) until viscous dissipation becomes important. The associated optimal forcing is streamwise rolls $\left(v^{\prime}, w^{\prime}\right.$ components) which result in growth of the streamwise velocity component (streaks).

In this paper, these characteristics of the lift-up mechanism, although re-framed for transport of high/low-speed fluid from the jet core/freestream to the jet freestream/core respectively, will be shown via global SPOD and resolvent analyses to be present in turbulent jets, expanding upon the work of Nogueira et al. (2019). We demonstrate the presence and energetic importance of the lift-up mechanisms over a range of Mach number, and characterize, as a function of frequency and azimuthal mode number, the interplay between the (now three) mechanisms (KH, Orr, lift-up). We will show that previously described experimental (Liepmann 1991; Paschereit et al. 1992; Liepmann \& Gharib 1992; Arnette et al. 1993; Citriniti \& George 2000; Alkislar et al. 2007; Cavalieri et al. 2013) and numerical (Martin \& Meiburg 1991; Caraballo et al. 2003; Freund \& Colonius 2009) data show the imprint of streaks, but had yet to be linked to the liftup mechanism. Here, resolvent analysis provides the key link between response modes 
observed in the SPOD spectrum and their underlying resolvent forcing modes, shedding light on the most active linear mechanisms throughout the wavenumber-frequency space.

The manuscript is organized as follows. In $\S 2$ we describe the databases and methods used in analysis. In $\S 3.1$ we introduce a collection of snapshots from the LES of a Mach 0.4 jet showing streaky characteristics. In $\S 3.2$ we present the dominant energies and gains computed using SPOD and resolvent analysis respectively. In $\S 3.3$ SPOD results approaching Strouhal number $S t \rightarrow 0$ and resolvent analysis at $S t=0$ are shown, identifying streaks and directly identifying the presence of the lift-up mechanism in turbulent jets. We then compare SPOD and resolvent modes in $\S 4$ at non-zero azimuthal wavenumbers and frequencies from $S t=0.6$ to $S t \rightarrow 0$ and present a sensitivity analysis delineating the various mechanisms pertaining to $\mathrm{KH}$, Orr, and lift-up, while also suggesting a mechanism map of the most amplified response mechanism throughout the wavenumber-frequency space for turbulent jets. We then conclude the manuscript in $\S 5$ addressing the presence of streaks throughout the domain, both near and far from the nozzle.

\section{Methods}

The LES database, resolvent analysis and SPOD were described in Schmidt et al. (2018) and Towne et al. (2018). For brevity, we recall the main details here.

\subsection{Large Eddy Simulation database}

The LES databases, including subsonic (Mach 0.4), transonic (Mach 0.9), and supersonic (Mach 1.5) cases, were computed using the flow solver "Charles" and details on numerical methods, meshing, and subgrid-models can be found in Brès et al. (2017); Brès et al. (2018) along with validation cases conducted at PPRIME Institute, Poitiers, France for the Mach 0.4 and 0.9 jets (Brès et al. 2018). The Mach 0.4 round jet, which is the jet investigated in detail in this manuscript, corresponds to a Reynolds number $R e_{j}=\rho_{j} U_{j} D / \mu_{j}=4.5 \times 10^{5}\left(M_{j}=0.9: R e_{j}=1.01 \times 10^{6}, M_{j}=1.5: R e_{j}=1.76 \times 10^{6}\right)$ where subscript $j$ indicates the value at the center of the jet, $\rho$ is density, $\mu$ is viscosity, and $M_{j}$ is the Mach number $M_{j}=U_{j} / a_{j}$, where $a_{j}$ is the speed of sound at the nozzle exit. The simulated jet corresponds to the experiments in Cavalieri et al. (2013); Jaunet et al. (2017); Nogueira et al. (2019) with the same nozzle geometry and similar boundary-layer properties at the nozzle exit.

Throughout the manuscript, variables are non-dimensionalized by the mean jet velocity $U_{j}$, jet diameter $D$, and pressure $\rho_{j} U_{j}^{2}$, with the resulting equation of state $p=\frac{\rho T}{\gamma M_{j}^{2}}$, with $T$ denoting temperature and $\gamma$ the ratio of specific heats. Frequencies are reported in Strouhal number, $S t=f D / U_{j}$, where $f$ is the frequency. The database consists of 10,000 snapshots separated by $\Delta t c_{\infty} / D=0.2\left(M_{j}=0.9: \Delta t c_{\infty} / D=0.2, M_{j}=1.5\right.$ : $\Delta t c_{\infty} / D=0.1$, where $c_{\infty}$ is the ambient speed of sound, and interpolated onto a structured cylindrical grid $x, r, \theta \in[0,30] \times[0,6] \times[0,2 \pi]$, where $x, r, \theta$ are streamwise, radial, and azimuthal coordinates,respectively. Variables are reported by the vector

$$
\boldsymbol{q}=\left[\rho, u_{x}, u_{r}, u_{\theta}, T\right]^{T},
$$

where $u_{x}, u_{r}, u_{\theta}$ are the three velocity components, and a standard Reynolds decomposition separates the vector into mean, $\overline{\boldsymbol{q}}$, and fluctuating, $\boldsymbol{q}^{\prime}$, components

$$
\boldsymbol{q}(x, r, \theta, t)=\overline{\boldsymbol{q}}(x, r, \theta)+\boldsymbol{q}^{\prime}(x, r, \theta, t) .
$$

Figure 1 displays one snapshot at the centerline of the streamwise velocity component, 


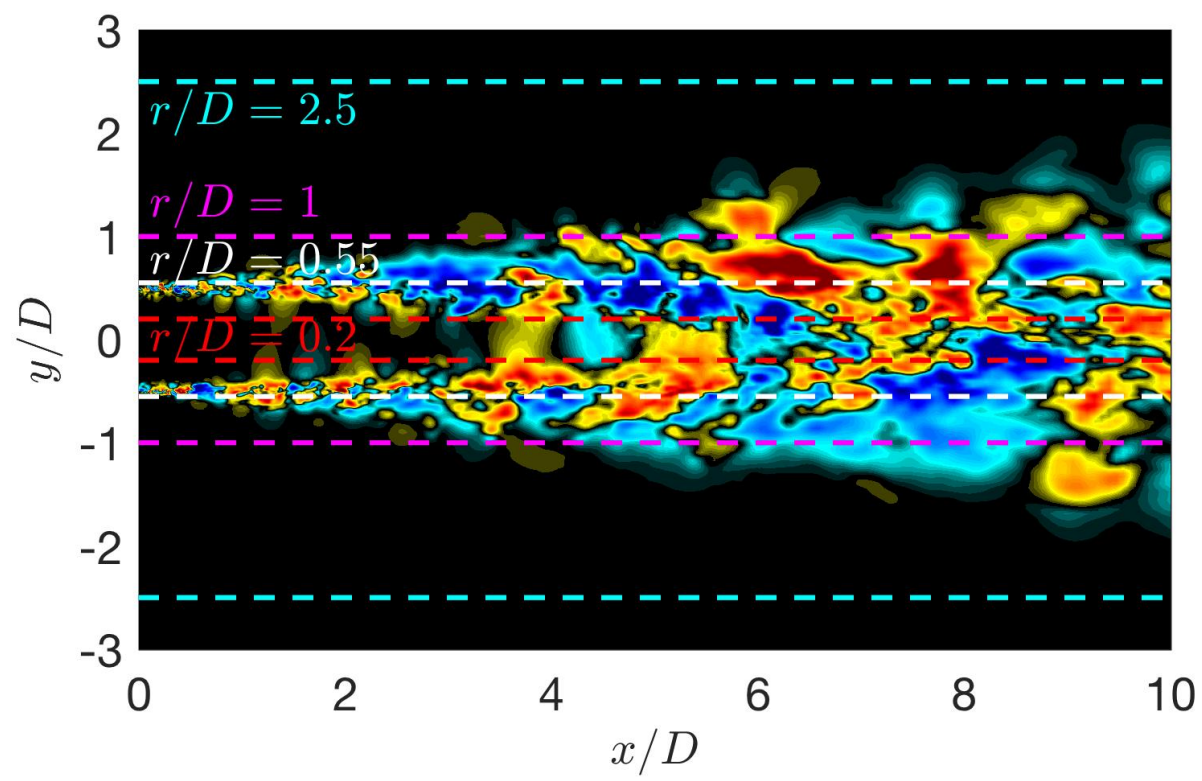

Figure 1: Large-Eddy Simulation snapshot of a centerline slice of fluctuating streamwise velocity. The lines show the areas of interest plotted in figure 2 .

where blue regions indicate fluctuations below the mean streamwise velocity and red indicate fluctuations above.

\subsection{Spectral Proper Orthogonal Decomposition}

Spectral proper orthogonal decomposition is used to determine an optimal set of orthogonal space-time correlated modes that describe the flow (Towne et al. 2018). Decomposing the LES database, $\mathcal{Q}$, in the azimuthal and temporal dimensions via the discrete Fourier transform gives the decomposed data matrices, $\mathcal{Q}_{m, \omega}$. The cross-spectral density tensor at a given frequency $\omega=2 \pi S t$ and azimuthal $m$ is then given by

$$
\mathbf{S}_{m, \omega}=\mathcal{Q}_{m, \omega} \mathcal{Q}_{m, \omega}^{H}
$$

and the SPOD eigenvalue problem presented by Lumley $(1967,1970)$ can be solved

$$
\mathbf{S}_{m, \omega} \mathbf{W} \boldsymbol{Q}_{s, m, \omega}=\boldsymbol{Q}_{s, m, \omega} \boldsymbol{\Lambda}_{m, \omega} .
$$

The SPOD modes are represented by the columns of $\boldsymbol{Q}_{s, m, \omega}$ (subscript $s$ denoting SPOD response mode matrix) and are ranked by the diagonal matrix of eigenvalues $\boldsymbol{\Lambda}_{m, \omega}=\operatorname{diag}\left(\lambda_{1}, \lambda_{2}, \ldots, \lambda_{N}\right)$. The modes are orthonormal in the norm $\boldsymbol{W}$, representing the compressible energy norm of Chu (1965)

$$
\left\langle\boldsymbol{q}_{1}, \boldsymbol{q}_{2}\right\rangle_{E}=\iiint \boldsymbol{q}_{1}^{*} \operatorname{diag}\left(\frac{\bar{T}}{\gamma \bar{\rho} M^{2}}, \bar{\rho}, \bar{\rho}, \bar{\rho}, \frac{\bar{\rho}}{\gamma(\gamma-1) \bar{T} M^{2}}\right) \boldsymbol{q}_{2} r d r d x d \theta
$$

and satisfy $\boldsymbol{Q}_{s, m, \omega}^{*} \boldsymbol{W} \boldsymbol{Q}_{s, m, \omega}=\boldsymbol{I}$.

Given SPOD is a discrete method for uncovering turbulent coherent structures, challenges persist for approaching the limit of $S t \rightarrow 0$. When performing SPOD a balance 
must be struck between snapshots per block (frequency resolution) and the number of blocks (convergence). To resolve frequencies over previous ranges of interest, $S t=[0.2,1]$ for the Mach 0.4 jet, block sizes of 256 snapshots with $50 \%$ overlap (resulting in 78 blocks) were used in Schmidt et al. (2018). In the case of uncovering stationary-in-time structures such as streaks, frequency resolution, and therefore block sizes, must be increased to approach the limit of $S t \rightarrow 0$. For the global SPOD analysis presented herein, and after experimentation with block sizes $(256,512,1024,2048)$ and overlap $(50 \%, 75 \%)$, we employ 1024 snapshots with $75 \%$ overlap (36 blocks) to attain the necessary frequency resolution, yet maintain convergence with sufficient blocks as $S t \rightarrow 0$. We also note that the use of 2048 snapshots per block produced almost identical SPOD modes as using 1024 snapshots, albeit with a greater uncertainty as the block ensemble was reduced to 16 blocks.

\subsection{Resolvent analysis}

For the round, statistically-stationary, turbulent jets we consider, the compressible Navier-Stokes, energy, and continuity equations are linearized through a standard Reynolds decomposition, and Fourier transformed both temporally and azimuthally to the compact expression

$$
\left(i \omega \mathbf{I}-\mathbf{A}_{m}\right) \boldsymbol{q}_{m, \omega}=\boldsymbol{f}_{m, \omega} .
$$

Here $\mathbf{A}_{m}$ is the discretized linear operator considering the mean field as the baseflow, $\boldsymbol{q}=\left[\rho^{\prime}, u_{x}^{\prime}, u_{r}^{\prime}, u_{\theta}^{\prime}, T^{\prime}\right]$ is the state vector, $\boldsymbol{f}$ constitutes the forcing in each variable.

The influence of viscosity in the linear operator, $\boldsymbol{A}_{m}$, has previously been based upon the molecular viscosity, $R e_{j}$, however, recent resolvent analyses incorporating an eddyviscosity model, Pickering et al. (2019) for turbulent jets and Morra et al. (2019) in channel flow, have shown substantial improvement for SPOD-resolvent comparisons. Thus, we proceed by including an eddy-viscosity model based upon the turbulent kinetic energy (TKE) suggested for turbulent jets by Pickering et al. (2019). This model was primarily chosen due to its simplicity and availability of the corresponding quantities from the LES database. The model takes the form

$$
\mu_{T}=\bar{\rho} c k^{1 / 2} l_{m},
$$

where $c$ is a scaling constant, $k$ is the mean flow turbulent kinetic energy, and $l_{m}$ is a chosen length scale representative of the mean shear layer thickness. $l_{m}$ is chosen as the width of the shear layer where the turbulent kinetic energy is more than $10 \%$ of its maximum value at each streamwise location, and the scaling constant $c=0.0065$ is used considering the favorable SPOD-resolvent alignments previously shown over $m=0-3$ and $S t=0.05-1$.

With the inclusion of an eddy-viscosity model the forward operator becomes $(i \omega \boldsymbol{I}-$ $\boldsymbol{A}_{m}-\boldsymbol{A}_{m, T}\left(\mu_{T}\right)$ ), where $\boldsymbol{A}_{m, T}\left(\mu_{T}\right)$ ) only possesses terms that include $\mu_{T}$ (equations for $\boldsymbol{A}_{m, T}\left(\mu_{T}\right)$ are included in Pickering et al. (2019)). The discretization scheme and boundary condition treatment are the same as those used in Schmidt et al. (2018) and refer the reader to that paper for further numerical details.

We can rewrite equation 2.6 by defining the resolvent operator, $\boldsymbol{R}_{\omega, m}=\left(i \omega \boldsymbol{I}-\boldsymbol{A}_{m}-\right.$ $\left.\boldsymbol{A}_{m, T}\left(\mu_{T}\right)\right)^{-1}$

$$
\boldsymbol{q}_{m, \omega}=\boldsymbol{R}_{m, \omega} \boldsymbol{f}_{m, \omega} .
$$

Introducing the compressible energy norm Chu (1965) via the matrix $\boldsymbol{W}$ to the forcing and response, where $\boldsymbol{W}=\boldsymbol{W}_{f}=\boldsymbol{W}_{q}$ gives the weighted resolvent operator

$$
\boldsymbol{H}_{m, \omega}=\boldsymbol{W}_{q}^{\frac{1}{2}} \boldsymbol{R}_{m, \omega} \boldsymbol{W}_{f}^{-\frac{1}{2}} .
$$


Taking the singular value decomposition of the modified resolvent operator gives

$$
\boldsymbol{H}_{m, \omega}=\boldsymbol{Q}_{m, \omega} \boldsymbol{\Sigma} \boldsymbol{F}_{m, \omega}^{H},
$$

where $\boldsymbol{Q}_{m, \omega}=\left[\boldsymbol{q}_{m, \omega}^{1}, \boldsymbol{q}_{m, \omega}^{2}, \ldots, \boldsymbol{q}_{m, \omega}^{N}\right]$ are the optimal response modes, $\boldsymbol{F}_{m, \omega}=$ $\left[\boldsymbol{f}_{m, \omega}^{1}, \boldsymbol{f}_{m, \omega}^{2}, \ldots, \boldsymbol{f}_{m, \omega}^{N}\right]$ are the optimal forcing modes, and $\boldsymbol{\Sigma}=\operatorname{diag}\left(\sigma_{1}, \sigma_{2}, \ldots, \sigma_{N}\right)$ are the gains. Accounting for the numerical quadrature and Chu energy weighting matrices normalize each forcing and response mode such that they are orthonormal and may be compared directly to their corresponding SPOD modes.

\subsection{Resolvent-based sensitivity analysis}

Since each resolvent mode may have contributions arising from different instability mechanisms, we aim to identify both the regions of the flow and components that drive optimal responses for each turbulent jet mechanism. As shown by Qadri \& Schmid (2017), a component-wise spatial sensitivity tensor can be calculated highlighting regions in the flow where small perturbations have a large effect on the resolvent gain and are influential to linear non-modal amplification of disturbances. The component-wise sensitivity tensor is computed as

$$
S_{i, j}=\sigma^{2} \operatorname{Re}\left(\boldsymbol{f}_{i} \circ \boldsymbol{q}_{j}^{*}\right),
$$

where subscripts $i, j$ denote the $i$-th or $j$-th component of the forcing or response, and $\circ$ denotes component-wise multiplication, giving the spatial sensitivity for each $i, j$ forcingresponse combination to the gain, $\sigma$. The sensitivity is calculated for the three forcing and response velocities of the momentum equation, presenting a $3 \times 3$ sensitivity tensor.

Further, we determine the relative, total sensitivity of each component, described by

$$
S_{i, j}^{\prime}=\frac{\left\|S_{i, j}\right\|_{F, \boldsymbol{W}}}{\sum_{i, j}\left\|S_{i, j}\right\|_{F, \boldsymbol{W}}} \times 100 \%,
$$

where $\|\cdot\|_{F, W}$ represents the weighted Frobenius norm by $\boldsymbol{W}$ and $S_{i, j}^{\prime}$ provides a percentage measure of the dominance of one forcing-response sensitivity to the overall sensitivity.

\section{Lift-up mechanism \& streaks}

For the remainder of this manuscript we report results for the Mach 0.4 turbulent jet. Similar analyses are provided for both the Mach 0.9 and 1.5 jets in the appendix.

\subsection{LES Visualization}

Previous SPOD and resolvent analysis of turbulent jets (Schmidt et al. 2018) showed that the energy of the most amplified mode increases for nonzero azimuthal wavenumber as $S t \rightarrow 0$. Therefore, considering significant energy is found at low frequencies, we expect to find elongated, streak-like structures to be visually present in the LES snapshots. Instantaneous snapshots are provided for four cylindrical surfaces at $r / D=0.2,0.55,1$, and 2.5. Figure 1 demonstrates where each surface is plotted from the LES snapshot and figure 2 shows the resulting unwrapped surfaces of fluctuating streamwise velocity, $u_{x}^{\prime}$. The figures are plotted with the circumferential location of the unwrapped surface, $r \theta / D$, as the y-axis (to maintain the physical aspect ratio of the data) against streamwise distance. Note that the plots are increasingly zoomed into the near nozzle region as the radial position is decreased.

These visualizations are analogous to experimental visualizations of Nogueira et al. 

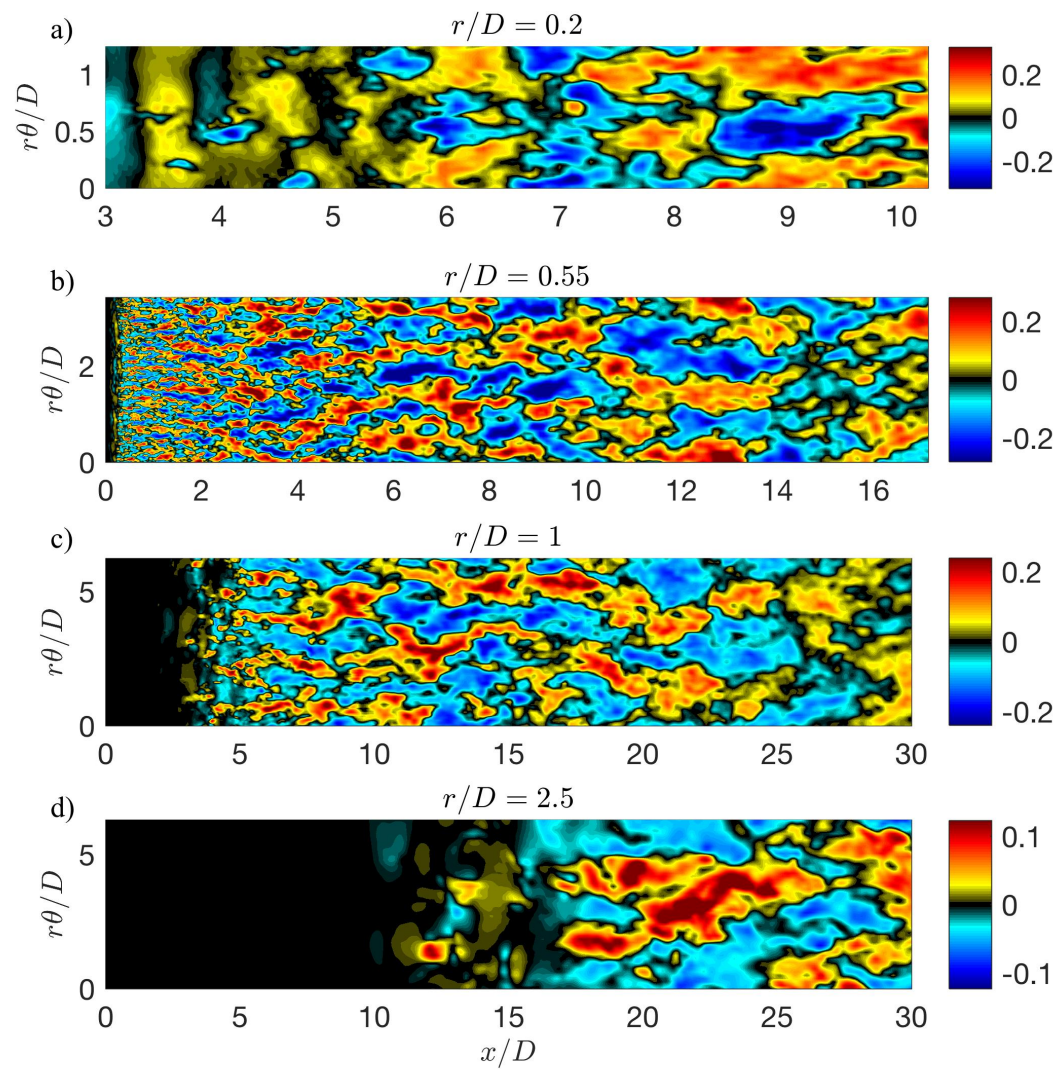

Figure 2: Instantaneous LES snapshots of streamwise fluctuating velocity, $u_{x}^{\prime}$, taken at radial locations $r / D=0.2,0.55,1,2.5$ with the y-axis representing the unwrapped surface $r \theta$. Only $2 / 5$ of the $r / D=2.5$ surface is shown in the last plot.

(2019) for a Mach 0.4 jet using Taylor's hypothesis. In their analysis, elongated streaky structures were found comparable to the LES visualizations for turbulent boundary layers of Eitel-Amor et al. (2014) using Taylor's hypothesis. They also noted how closely their structures possessed similar qualities to streaky structures found in channel flow (Monty et al. 2007), pipe flow (Hellström et al. 2011), and boundary layers (Hutchins \& Marusic 2007). The visualizations presented here show similar characteristics of streaky behavior, but do not use Taylor's hypothesis and instead present instantaneous snapshots of the flow.

Beginning with radial surface $r / D=0.2$ we attain a viewpoint from within the potential core. For $x / D=[3,6]$ structures appear to have a dominant $r \theta / D$ dimension and may be associated to $\mathrm{KH} m=0,1$ instabilities (i.e. non-streaky) extending into the potential core. At $x / D \approx 6$, where the potential core ends, the turbulent kinetic energy increases and streamwise elongated structures begin to appear; they become more pronounced further downstream. For the radial surface $r / D=0.55$, we see streaky structures of smaller scale close to the nozzzle, and larger scale further downstream, are similar to the structures visualized by Nogueira et al. (2019) at this radial location. These structures also meander as they propagate downstream, a quality also observed 

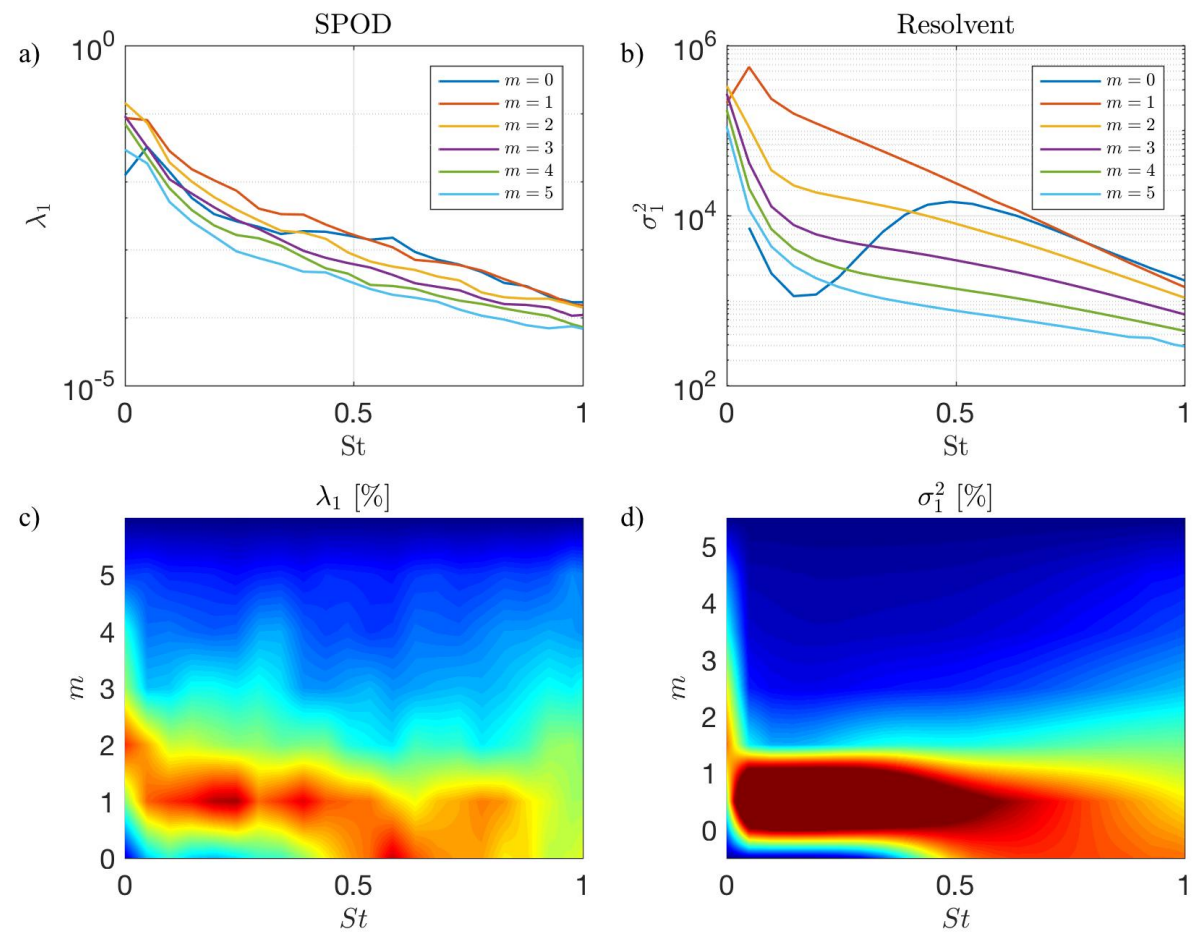

Figure 3: Energy of the most amplified mode from SPOD (left) and resolvent (right) analyses. The top row $(a, b)$ displays the energies and gains for the first six azimuthal wavenumbers. The bottom row $(\mathrm{c}, \mathrm{d})$ recasts the plots as a percentage of the sum of energy at each azimuthal wavenumber with contours ranging from $0-40 \%$.

in boundary layer flows by Hutchins \& Marusic (2007). Comparable behavior is seen for both $r / D=1$ and 2.5. In each, one may note that directly prior to the emergence of streamwise elongated structures (once the surface crosses into the turbulent region) faint structures elongated in the circumferential direction are detected, likely related to high-frequency, $m=0,1 \mathrm{KH}$ signatures.

\subsection{SPOD and Resolvent Analysis}

SPOD (using the parameters of Schmidt et al. (2018)) and resolvent analyses were performed over a range of Strouhal frequencies and azimuthal wavenumbers. Figures $3(\mathrm{a}, \mathrm{b})$ show the $\operatorname{SPOD}\left(E_{m, S t}^{S P O D} \equiv \lambda_{m, S t}\right)$ and resolvent $\left(E_{m, S t}^{\text {resolvent }} \equiv \sigma_{m, S t}^{2}\right)$ energies/amplifications of the leading mode. In figures $3(\mathrm{c}, \mathrm{d})$ the same data are normalized at each frequency with the sum of energy over all azimuthal wavenumbers $\left(E_{m, S t} / \sum_{m} E_{m, S t} \times 100\right)$ and plotted in the $m-S t$ plane. This highlights the dominant wavenumbers at each frequency and facilitates the identification of different instabilities. The map is interpolated for non-integer wavenumbers despite their discrete nature. This representation was chosen because we found it easier to visually interpret the trends, and to more readily compare it to wavenumber-frequency maps familiar from boundary layer flows where wavenumber is continuous (Monokrousos et al. 2010). For reference the semi-discrete representation of figure 3 is provided in the appendix.) 

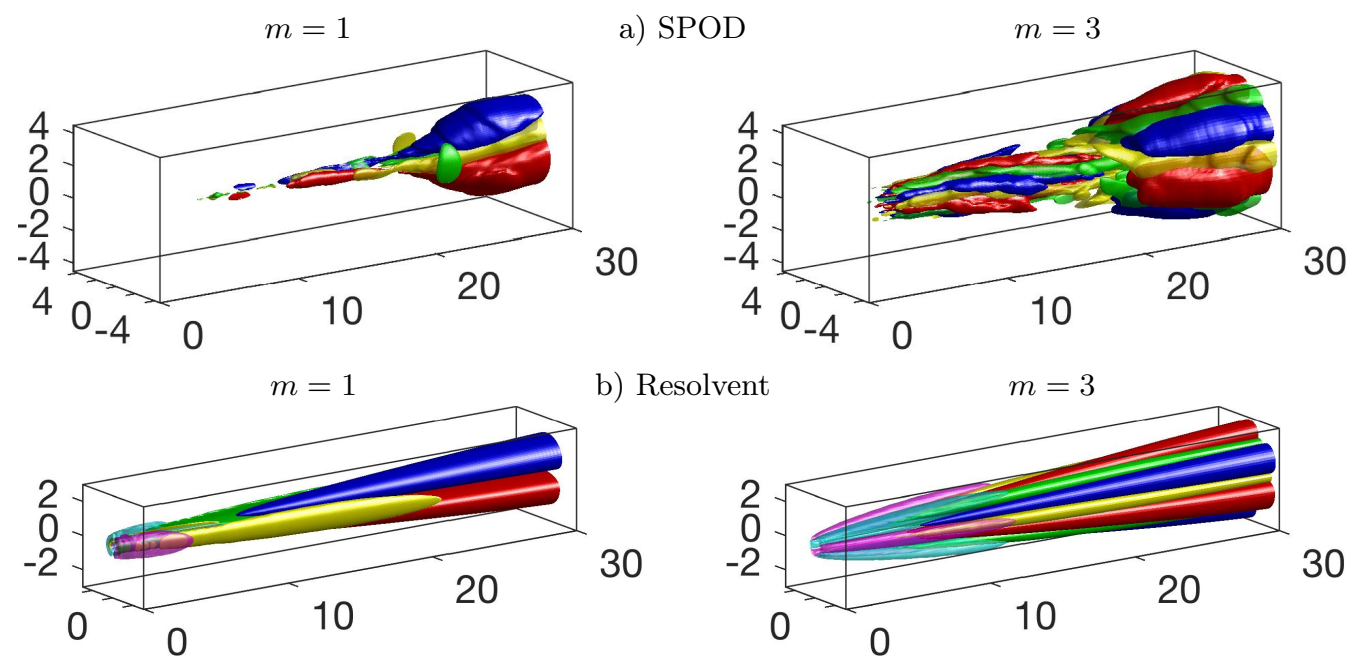

b) Resolvent $m=3$

Figure 4: Streaks deduced via SPOD and resolvent analyses for $m=1$ and 3. a) Most energetic SPOD mode at $S t \rightarrow 0$ for $m=1$ (left) and $m=3$ (right). Isosurfaces of streamwise velocity (red, blue) and streamwise vorticity (yellow, green), both at $\pm 25 \%$ of their maximum value. b) Global resolvent forcing and response for $m=1$ (left) and $m=3$, (right) at $S t=0$. The streamwise forcing vorticity is shown in magenta-cyan with isosurfaces $\pm 0.05\left\|f_{\omega_{x}}\right\|_{\infty}$ for $m=1$ and $\pm 0.15\left\|f_{\omega_{x}}\right\|_{\infty}$ for $m=3$, streamwise response vorticity is shown in yellow-green with isosurfaces $\pm 0.5\left\|q_{\omega_{x}}\right\|_{\infty}$, and streamwise response velocity is shown in red-blue with isosurfaces $\pm 0.25\left\|q_{u_{x}}\right\|_{\infty}$.

There is a qualitative agreement between the SPOD and resolvent energy content. There are two overlapping regions in $m-S t$ space where there exist energetic structures. The region near $S t=0.6$ at low azimuthal wavenumbers (particularly $m=0$ ) is associated with the KH mechanism At low frequencies, meaning $S t \rightarrow 0$, the $m=0$ mode has an anomalous behavior, with the dominant mode switching from $\mathrm{KH}$ to Orr mechanism for $S t<0.2$ (Schmidt et al. 2018). At low frequencies and mainly for $m=1$ and 2, a second energetic region is observed. This region comprises the lift-up mechanism that is described more fully in what follows. In the wavenumber-frequency contour plots, these trends resemble those obtained for laminar boundary layers (Monokrousos et al. 2010), where lift-up dominates as streamwise wavenumbers approach 0, or equivalently the frequency approaches zero for spatially developing flows. For nonzero azimuthal wavenumbers, where the lift-up mechanism is expected to be found due to its threedimensional characteristics, $m=1$ provides the largest energy across all frequencies, save $m=2$ at $S t \approx 0$, followed by a gradual reduction for higher azimuthal wavenumbers.

\subsection{Global characteristics of streaks}

The SPOD modes for $S t \rightarrow 0$ are shown in figure 4, calculated using 1024 snapshots and $75 \%$ overlap (36 blocks). The streaks are visualized and identified in physical space based on the reconstructed streamwise perturbation velocity:

$$
u_{x}^{\prime}(x, r, \theta)=\operatorname{Re}\left(u_{x_{m}}^{\prime}(x, r) \exp (-i m \theta)\right) .
$$

For $m=1$, a well-defined streak is observed throughout the domain starting close to the end of the potential core. In the $m=3$ plot, streaks are seen upstream and downstream of the potential core, however, at about $x / D=[15,20]$ the streaks are less dominant 


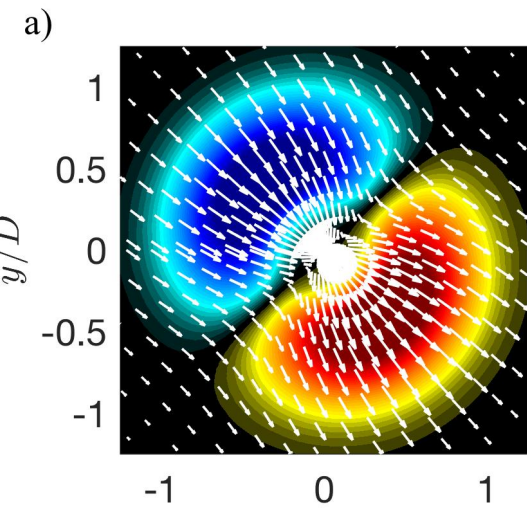

b)
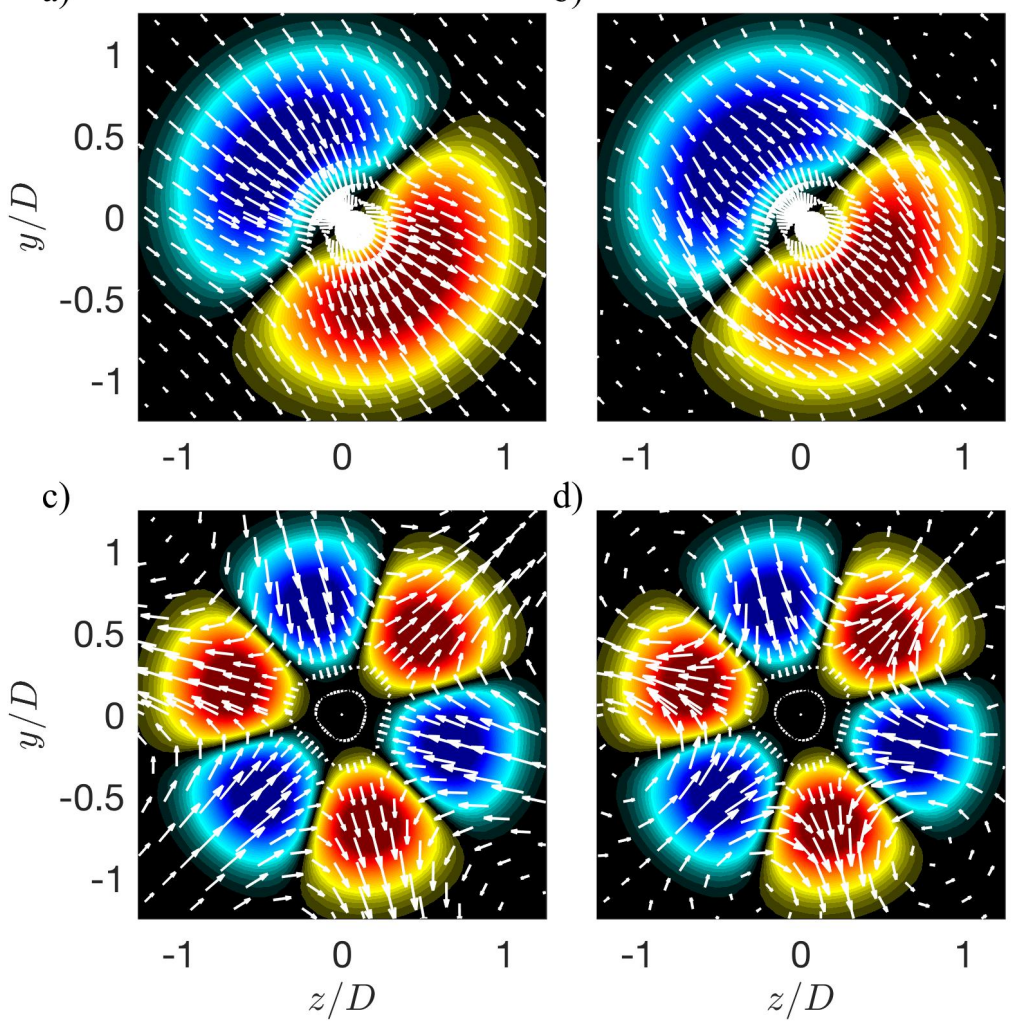

d)

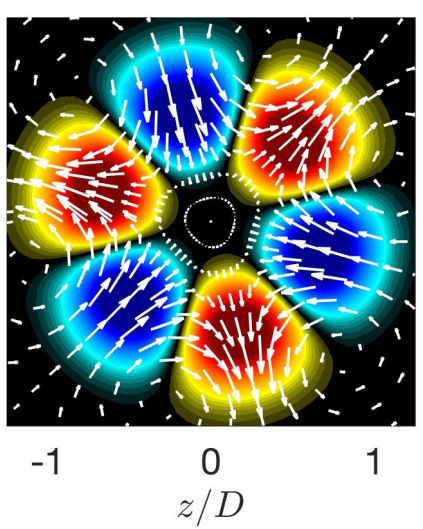

Figure 5: Cross plane at $x / D=5$ of rolls and streaks computed via resolvent in figure 4. The contours for (a-d) present streaks with values set at $\pm 0.75\left\|q_{u_{x}}(x / D=5)\right\|_{\infty}$, red and blue respectively. (a,b) and (c,d) correspond to $m=1$ and $m=3$ respectively, while the overlaid vectors represent the forcing rolls in $(\mathrm{a}, \mathrm{c})$ and response rolls in $(\mathrm{b}, \mathrm{d})$.

and appear to slightly rotate. The slight rotation is likely an artifact of imperfect SPOD convergence at $S t \rightarrow 0$, as approaching zero frequency still includes small, but non-zero, frequencies. Interestingly, the slight rotation of these modes are rather similar to energetic POD modes found by Freund \& Colonius (2009).

Further evidence these structures are due to the lift-up mechanism is shown by the yellow-green isosurfaces of streamwise vorticity included in figure 4 . The presence of streamwise vorticity, or rolls, and their particular location, situated precisely between positive and negative streamwise velocity contours, are indicative of the lift-up mechanism. The observation of such streamwise vortices in jets has previously been reported by a number of authors, Liepmann (1991); Martin \& Meiburg (1991); Paschereit et al. (1992); Liepmann \& Gharib (1992); Arnette et al. (1993); Alkislar et al. (2007), while both Citriniti \& George (2000) and Caraballo et al. (2003) noted that large streamwise velocity accompanied such vortices. Given our current observations, and those of resolvent analysis to follow, we suggest these streamwise vortices previously observed are linked to the lift-up mechanism.

Performing a global resolvent analysis $m=1,3$ at $S t=0$ allows for further understanding of the forcing mechanisms which give rise to the observed behavior of the SPOD modes. We begin by comparing response mode shapes of SPOD and resolvent analyses, 
as well as the associated resolvent forcing, in figure 4 for both azimuthal wavenumbers. In each figure we see the canonical lift-up progression. The optimal forcing acts on the crossstream components upstream at the nozzle, with associated streamwise vorticity $f_{\omega_{x}}$, in order to optimally generate rolls, $\omega_{x}$. The rolls, in turn, give rise to streamwise velocity responses, $u_{x}$, i.e. streaks. The spatial location of the forcing and responses are correctly inline with the description of the lift-up mechanism in wall bounded flows (Monokrousos et al. 2010). Further, the responses in both streamwise vorticity and velocity shown by the SPOD modes in figure 4 agree quite well with the resolvent findings for both azimuthal wavenumbers.

Although the 3D representation of figure 4 presents many of the salient characteristics of the lift-up mechanism, a $2 \mathrm{D}$ cut at $x / D=5$ shown in figure 5 highlights the radial and azimuthal velocity components of forcing and response rolls with respect to streaks. In figure $5(\mathrm{a}, \mathrm{c})$ the forcing velocity vectors show the lifting of high/low-speed fluid from the center/outer jet inducing positive/negative streaks, respectively. More precisely, the forcing rolls show strong radial velocities coincident with maximum streamwise response for both azimuthal wavenumbers, while strictly azimuthal velocities are located exactly between positive and negative streaks. It is worth noting that this observation has implications to results found via the resolvent sensitivity analysis and will be discussed further in $\S 4$.

Additionally, the forcing rolls give rise to response rolls shown in figure $5(\mathrm{~b}, \mathrm{~d})$ presenting similar velocity characteristics as the forcing vectors, however, the response vectors show the vortices are centered near the jet, a quality not shared with the forcing vectors. Again, it is these response vortices that are likely the components of the streamwise vortices observed by the numerous authors referenced earlier.

Figure 6 shows quantitative comparisons between the compressible energy inner product computed at each streamwise position for SPOD $(S t \rightarrow 0)$ and resolvent $(S t=0)$, for the first 5 resolvent/SPOD modes. It is worth noting that the full compressible energy inner product of the 5 variables over the streamwise direction is unity by construction. The curves from SPOD and resolvent are trend-wise similar, with noise in the SPOD results due to statistical convergence issues. The streamwise velocity response (i.e. streaks) is clearly the dominant response variable throughout the domain for both the resolvent and SPOD analyses. For the forcing terms, the radial and azimuthal velocities dominate throughout the domain by two orders of magnitude and correspond to the streamwise vorticity (i.e. rolls). Both the forcing and response energy curve observations provide strong evidence of the lift-up mechanism.

The streaky structures identified above are reminiscent of the dominant kineticenergy-based structures educed by Freund \& Colonius (2009) using snapshot POD from numerical simulations and by Citriniti \& George (2000) performing local SPOD (from a hot-wire array) three diameters downstream of a round jet nozzle, but they had not been linked to the lift-up mechanism in the previous studies. Based on global energy, we find that $m=1$ is the most amplified azimuthal wavenumber, with spatial development downstream of the potential core where the shear layers merge. Later, in $\S 5$ we show that streaks of higher azimuthal wavenumbers are also present near the nozzle, but they are considerably less energetic such that they can only be identified with localized analysis $(\S 5)$.

The generation of streaks through the lift-up mechanism is also observed for the transonic, $M=0.9$, and supersonic, $M=1.5$ cases, showing similar trends. SPOD and resolvent analyses results for both additional jets are shown in appendix A. 

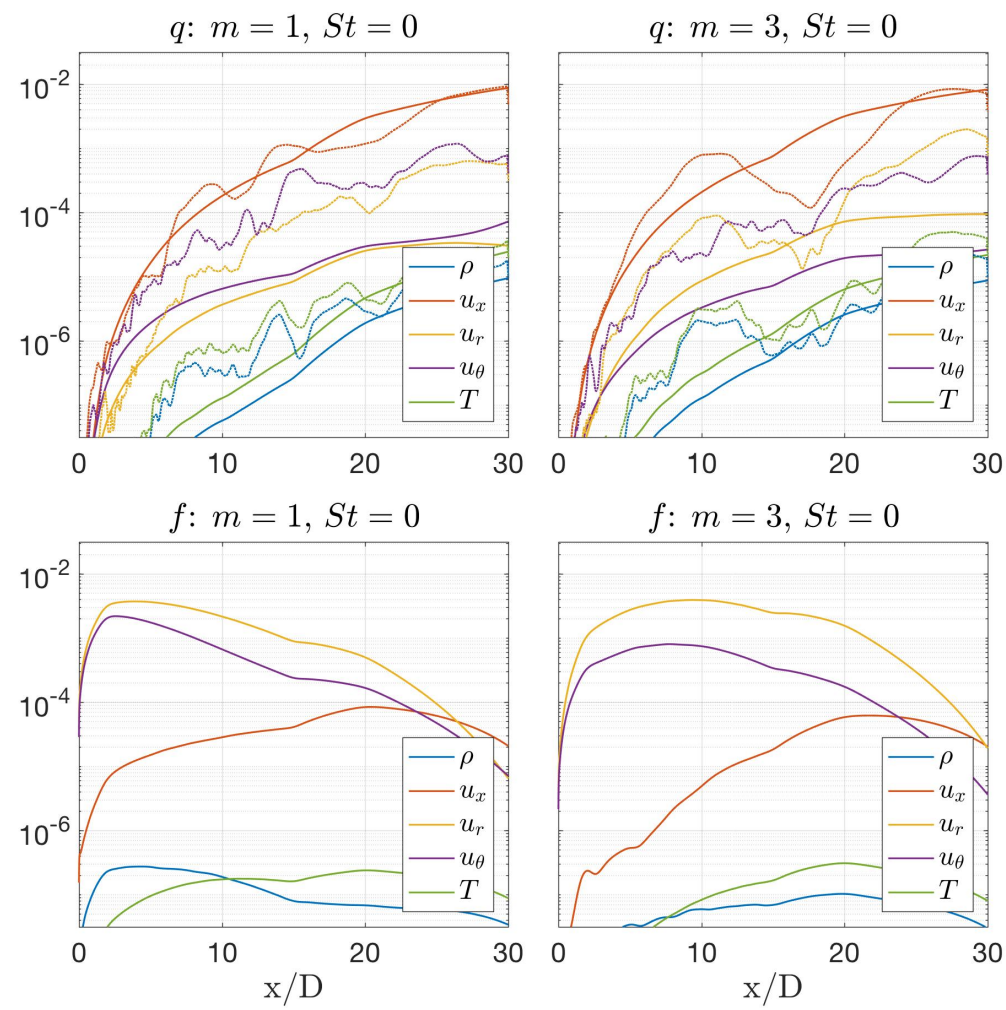

Figure 6: Component-wise energy evolution in the streamwise direction of the streaks for $S t=0$ and $m=1$ and 3. Optimal response (top) and forcing (bottom) energy from resolvent (solid) and SPOD (dotted) analyses as a function of streamwise coordinate from the nozzle exit.

\section{Interplay of lift-up, Kelvin-Helmholtz, and Orr mechanisms}

Figure 7 a) summarizes the regions of dominance between the $\mathrm{KH}$, Orr, and lift-up mechanisms as a function of frequency and azimuthal mode number. $\mathrm{KH}$ is dominant along the frequency axis (i.e. $m=0$ ) for $S t>0.3$, while lift-up is dominant along the azimuthal wavenumber axis $(S t=0)$ for $m>0$. Orr, on the other hand, is always present and appears as the dominant mechanism only when $\mathrm{KH}$ is not dominant $(S t<0.3)$ and lift-up is absent $(m=0)$. To further elucidate the physics of these mechanisms, we compare their SPOD and resolvent representations at the specific wavenumber-frequency pairs (combinations of $m=[0,1,3]$ and $S t=[0.05,0.2,0.6]$ denoted by white circles on the figure.

Figure 7 shows the streamwise velocity of the most energetic/amplified SPOD (b) and resolvent response (c) modes and resolvent forcing (d) modes for these 9 azimuthal wavenumber-frequency pairs. Figures 8 and 9 show the associated radially integrated energy plots for SPOD and resolvent response modes and resolvent forcing modes, respectively. Finally, sensitivity of the resolvent gain with respect to forcing and response variables plotted for selected cases associated with $\mathrm{KH}$, Orr, and lift-up mechanisms in figure 10 .

These plots are analyzed in what follows below. Overall, one can see that the resolvent 
a) Cartoon mechanism map

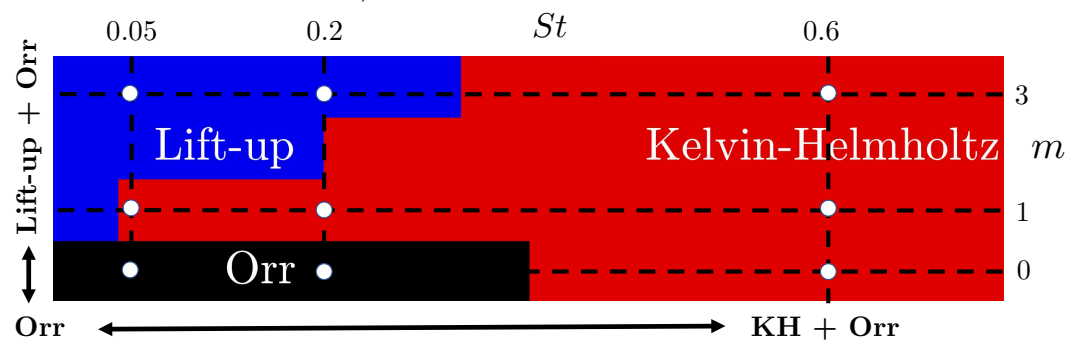

b) SPOD

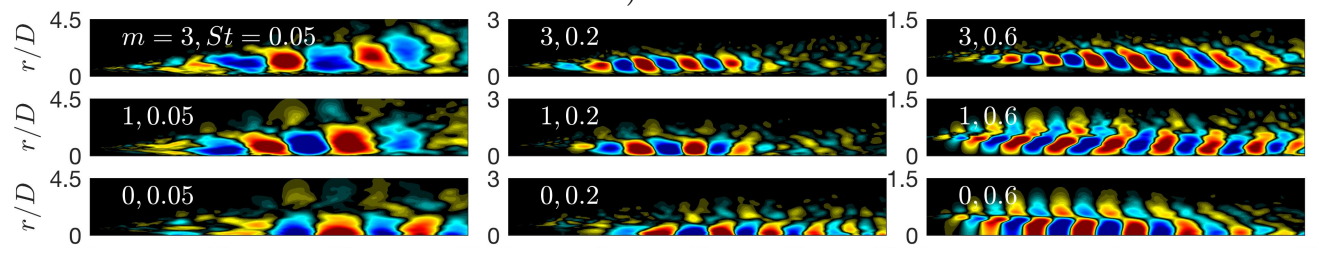

c) Resolvent response
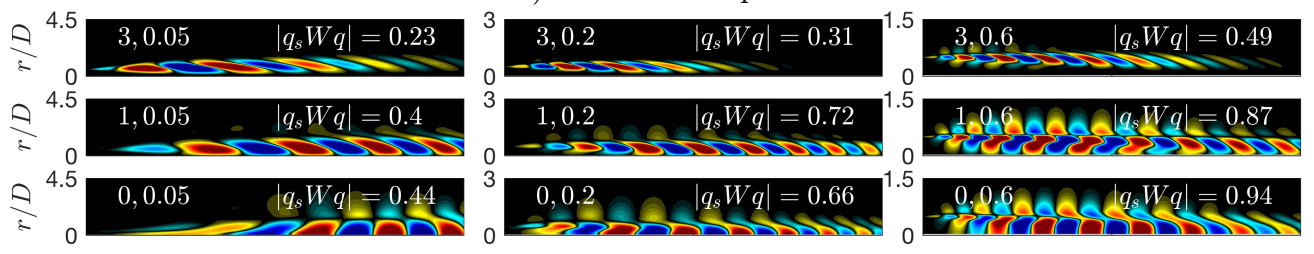

d) Resolvent forcing
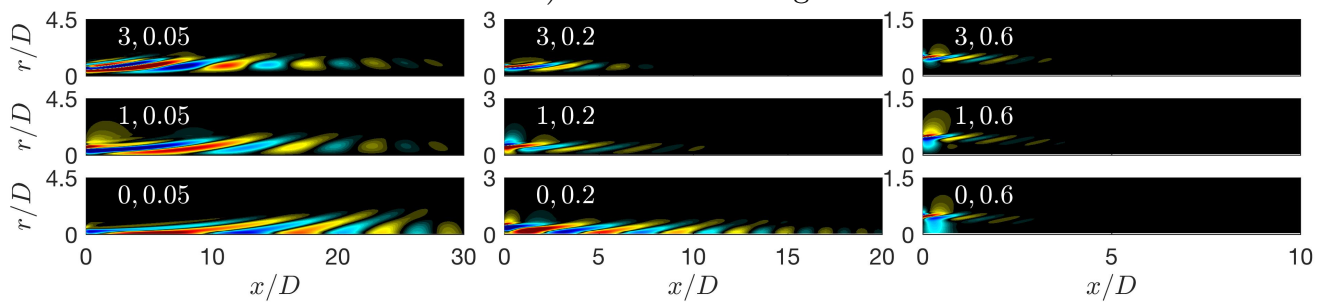

Figure 7: Cartoon mechanism map (a), most energetic SPOD (b), resolvent response (c), and resolvent forcing (d) modes for azimuthal wavenumbers $m=[0,1,3]$ and frequencies $S t=[0.05,0.2,0.6]$. Streamwise velocity perturbation, $u_{x}^{\prime}$, component is shown with contours corresponding to $\pm 0.5\left\|u_{x}^{\prime}\right\|_{\infty}$, with projection coefficients, $\left|q_{s} W q\right|$, between the full SPOD responses, $q_{s}$, and resolvent responses, $q$, provided in c). The locations of the white circles mirror the placement of the subsequent SPOD and resolvent analyses and denote their location in the wavenumber-frequency space.

and SPOD analyses show significant agreement between the mode shapes and their relative energy content, particularly for higher frequencies where $\mathrm{KH}$ low-rank behavior is exhibited.

\subsection{Kelvin-Helmholtz $+\operatorname{Orr}(S t=0.6, m=0,1)$}

At $[m, S t]=[0,0.6]$, the response is dominated by the $\mathrm{KH}$ mode, which is spatially unstable near the nozzle. These modes and their low-rank behaviour are also described in Schmidt et al. (2018), with the exception that here the streamwise velocity component 

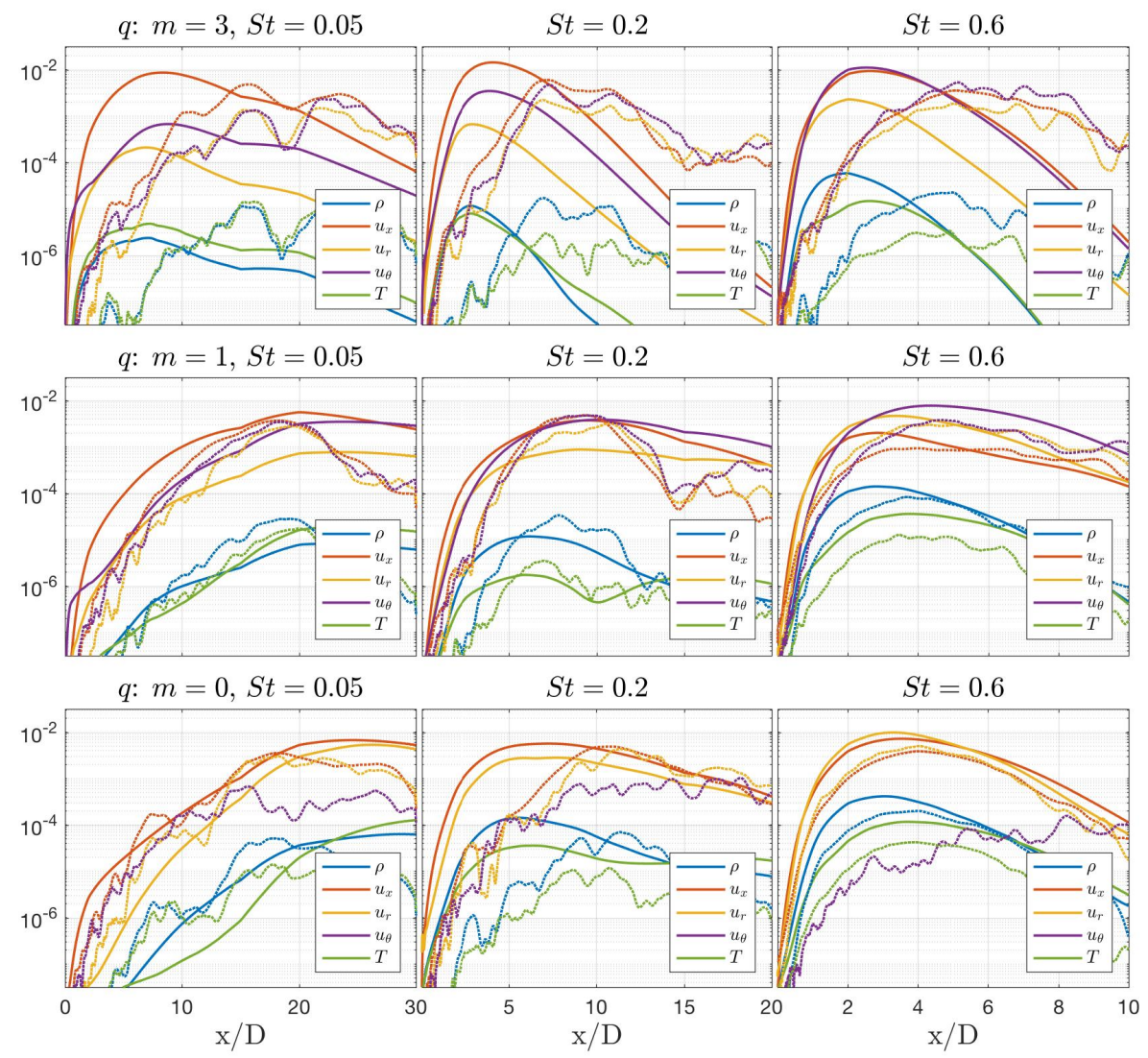

Figure 8: Streamwise evolution of component-wise energy for resolvent (solid lines) and SPOD (dotted lines) analyses. The layout of the figure mirrors that of figure 7 with $m=3$ represented in the first row followed by $m=1,0$, and the first column displaying the lowest $S t$ increasing with columns to the right. Note that the truncated domains shown in figure 7 are maintained for each $m-S t$ pair.

is shown rather than the pressure component. The structures near the nozzle are tilted at an angle $\sim 45^{\circ}$ and within a diameter they become vertically aligned, and there is a 90 degree phase shift at a critical layer where the apparent phase speed is equal to the mean flow speed $\dagger$. These observations provide evidence of a $\mathrm{KH}$ response. Once the $\mathrm{KH}$ mode saturates, approximately 5 diameters downstream of the nozzle, the wavepacket continues to tilt, reaching finally an angle of $-45^{\circ}$. The tilting phases are evidence of the Orr mechanism (Tissot et al. 2017). Similar behavior is observed for $m=1$ wavenumbers at $S t=0.6$, for which the $\mathrm{KH}$ response is also dominant.

The energy curves for $[m, S t]=[0,0.6]$ are also reminiscent of $\mathrm{KH}$ instability with rapid, exponential growth, dominated by streamwise and radial velocity, starting from the nozzle exit followed by saturation and decay by $x / D \approx 5$. For nonzero azimuthal wavenumbers, the azimuthal perturbation velocity is increasingly important. At this frequency, forcing modes for all azimuthal wavenumbers are concentrated upstream with

$\dagger$ For global modes, the phase speed is estimated using the procedure in Schmidt et al. (2018) 

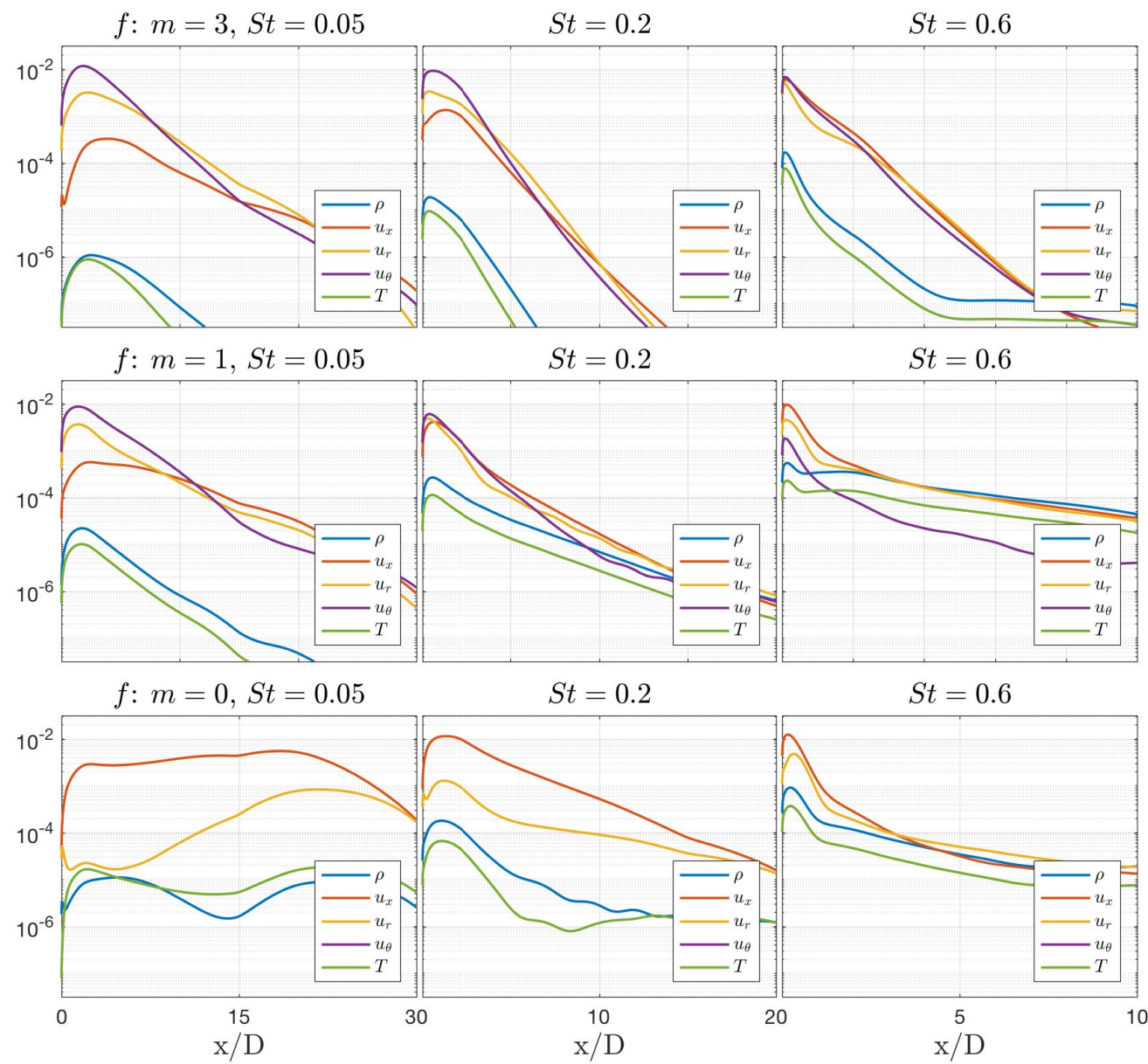

Figure 9: Streamwise evolution of component-wise energy for resolvent forcing modes. The layout and streamwise extent of each plot is identical to figure 8 .

the maximum forcing energy occurring within $x / D \leqslant 0.1$ along the lip line. The forcing decays by at least two orders of magnitude within the first two jet diameters. Although the dominant response is $\mathrm{KH}$ for this frequency the shape for each of the forcing modes are Orr-type, where structures lean against the direction of mean shear by an angle of $\sim 45^{\circ}$ and are concentrated along the critical layer (Tissot et al. 2017). Meanwhile the gain sensitivity (figure $10 \mathrm{a}$ ) ) is localized to the inner edge of the shear layer terminating around the close of the potential core, with similar sensitivity to both nonzero velocity components and their forcings. This is in contrast with the sensitivity map associated with the Orr and lift-up mechanisms discussed next.

\section{2. $\operatorname{Orr}(S t \rightarrow 0, m=0)$}

For a fixed domain size, we expect the dominance of the Orr mechanism as the frequency of the axisymmetric mode, $m=0$, is reduced. Decreasing the frequency, the $\mathrm{KH}$ mode becomes less unstable and the maximum amplification region moves further 
downstream. This behavior is observed as the frequency is reduced to $S t=0.2$, and further to $S t=0.05$, for $m=0$.

At $[m, S t]=[0,0.05]$ the Orr mechanism is dominant. The response modes are spatially distributed and possess a continuous turning from $-45^{\circ}$ to $+45^{\circ}$ throughout their envelope. We still observe a phase change across the critical layer, however this behavior is rather weak when comparing to the $\mathrm{KH}$ dominated modes described above $(S t=0.6)$. By contrast with $\mathrm{KH}$, the response is less strongly amplified, peaking some 20 diameters downstream and only weakly damped thereafter. The forcing is entirely dominated by streamwise velocity, and is spatially distributed; for $m=0$ and $S t=0.05$ it has nearly constant amplitude up to $x / D=20$. Finally, the sensitivity map (figure $10 \mathrm{~b}$ )) shows high sensitivity far downstream and near the centerline, and is dominated by a sensitivity of the streamwise velocity to forcing of the streamwise velocity. Again, this behavior can be contrasted with the lift-up mechanism discussed next.

$$
\text { 4.3. } \quad \text { Lift-up }+\operatorname{Orr}(S t \rightarrow 0, m>0)
$$

For $m \neq 0$, as we approach zero frequency, the wavepackets show a different structure from the $\mathrm{KH}$ dominated regimes. While the response mode structures are tilted at $+45^{\circ}$ and the forcing modes are tilted at $-45^{\circ}$, as they are for the Orr mechanism, the energy curves provide evidence of lift-up. For $[m, S t]=[3,0.05]$ the streamwise velocity is, by approximately an order of magnitude, larger than the other components, whereas for $[m, S t]=[0,0.05]$ (i.e. Orr only) both streamwise and radial velocities contribute similarly to the total mode energy. The significance of additional streamwise velocity energy content is directly associated with the presence of streaks. The forcing, by contrast, shows a dominance of radial and azimuthal velocities, producing streamwise vorticity. In tandem, these observations describe the lifting of fluid, via cross-plane rolls, from highand low-speed regions of the jet to the fluctuating streamwise-velocity response.

The lift-up mechanism persists to nonzero frequencies, where the streaks are not stationary, and rotate about the jet. Evidence of streaks are seen for all nonzero wavenumbers up to frequencies of $S t \approx 0.2$. As the streaks slowly rotate about the jet, their intersection with a 2D plane presents mode shapes similar to the Orr-type mechanism, which may explain why they were not observed in earlier studies.

Further evidence for the lift-up mechanism is given by the sensitivity map (figure 10 c) ). Unlike $\mathrm{KH}$ and Orr, the largest sensitivity is found in radial forcing to streamwise response along with a spatial region of sensitivity that is unique when compared to $\mathrm{KH}$ and Orr. The shift of sensitivity to the radial-forcing, streamwise-response pair, is expected for the lift-up mechanism as the radial forcing component is responsible for lifting high- and low-speed regions of the jet to the streamwise response. The sensitivity also has a varied spatial location and is found lifted off the centerline, in both upstream and downstream locations. Together, these two observations indicate the lift-up mechanism is a dominant mechanism found in turbulent jets, providing significant contributions to the perturbation energy of the flow at low frequency, non-zero azimuthal wavenumbers.

One additional, and surprising, result of the sensitivity analysis for lift-up is the lack of sensitivity for the streamwise response to the azimuthal forcing, a forcing component that is necessary for the presence of forcing vortices. The large sensitivity to the radial forcing and zero sensitivity in the azimuthal forcing may be explained by the azimuthal location of the radial and azimuthal forcing components with respect to the streaks. Sensitivity to the gain is defined as the real of the outer product of the forcing and response, effectively presenting largest sensitivities for forcings and responses which are coincident in space. This is observed in figure $5(\mathrm{a}, \mathrm{c})$ in $\S 3.3$ where the largest regions of radial 
a) $m=0, S t=0.6 . \mathrm{KH}$ dominated.

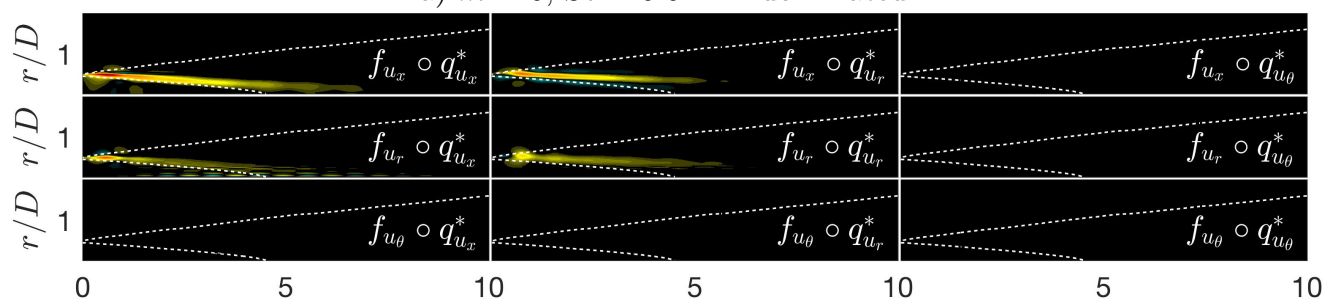

b) $m=0, S t=0.05$. Orr dominated.

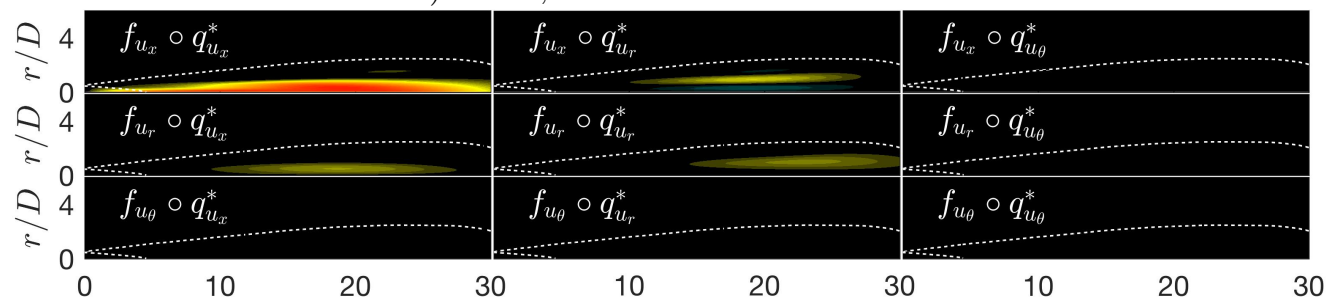

c) $m=3, S t=0$. Lift-up dominated.

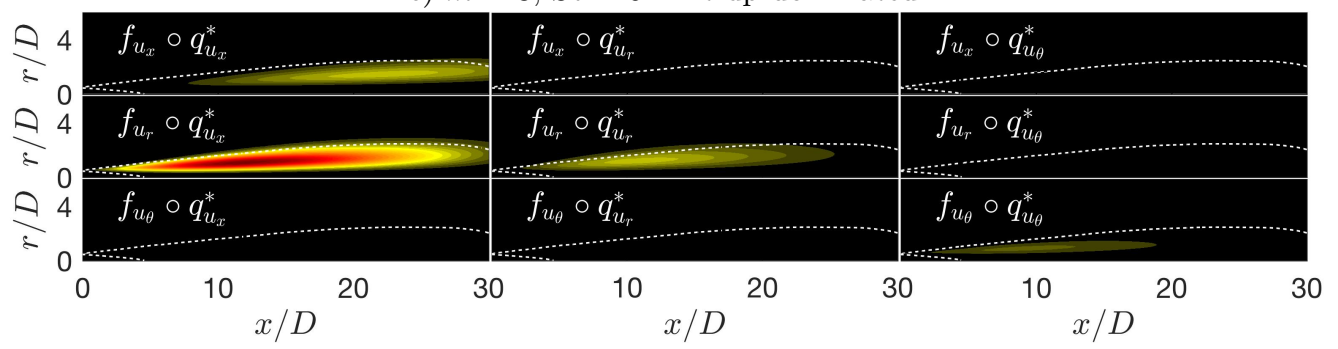

Figure 10: Resolvent-based spatial sensitivity plots for all 9 velocity combinations for $[m, S t]=[0,0.6](\mathrm{a}),[m, S t]=[0,0.05](\mathrm{b})$, and $[m, S t]=[3,0](\mathrm{c})$. Contours are set for each of the three plots by the maximum sensitivity, $\pm\left\|f \circ q^{*}\right\|_{\infty}$, across all 9 velocity pairs. Note for the $\mathrm{KH}$ case the domain is reduced to $x / D=[0,15]$ and $r / D=[0,2]$ to highlight the upstream behavior, as no sensitivity is observed outside this domain. The white, dotted lines provide reference to the envelope of the jet that is $>10 \%$ of the maximum turbulent kinetic energy.

forcing are found to be coincident with the most energetic regions of the streaks (i.e. large sensitivity), while the greatest azimuthal forcing regions are found where streaks have zero amplitude (i.e. zero sensitivity). An analogous result, in terms of coincidence of forcing and response, is seen for the $\mathrm{KH}$ sensitivity in figure $10 \mathrm{a}$ ). The $\mathrm{KH}$ response extends many diameters downstream, however, the sensitivity is localized along the potential core where both the forcing, located near the nozzle and along the shear layer, and response are active. Overall, the sensitivity analysis to the resolvent gain provides a useful tool for identifying mechanisms by distilling both the most important forcing components and their spatial properties.

\subsection{Mechanism Map}

Following identification of the salient properties of each turbulent jet mechanism, we propose the the mechanism map shown in figure 11, estimating the regions of mechanism dominance in the wavenumber-frequency space for turbulent jets. The mechanism map is 


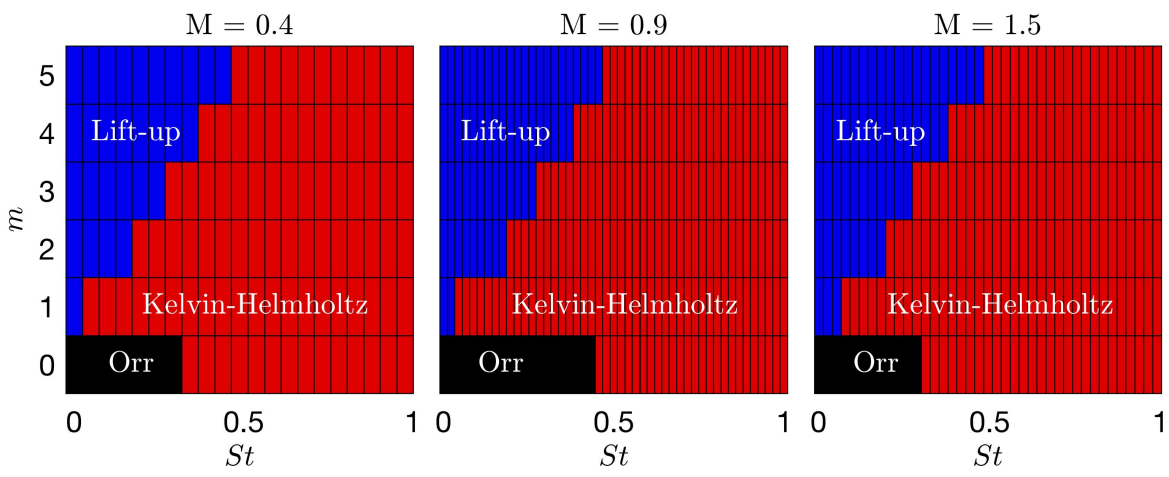

Figure 11: Mechanism map estimating the dominant mechanisms in the wavenumberfrequency space of the most amplified resolvent response for turbulent jets. Black, red, and blue represent the Orr, KH, and lift-up mechanisms, respectively.

determined through metrics of the relative sensitivity, $S_{i, j}^{\prime}$, assessing the overall influence one forcing-response pair has on the total sensitivity. From figure 10 b) and c) we see that for Orr dominated responses the primary sensitivity is from the $f_{u_{x}} \circ q_{u_{x}}$ pair, while lift-up has primary sensitivity in $f_{u_{r}} \circ q_{u_{x}}$. KH dominated responses, on the contrary, show an even mix of relative sensitivity between forcing-response pairs. Considering these observations two metrics are defined, lift-up is determined to be dominant when $S_{u_{r}, u_{x}}^{\prime}>$ $30 \%$ and Orr is dominant when $S_{u_{x}, u_{x}}^{\prime}>55 \%$.

The resulting map in figure 11 presents a rather consistent estimate of the regions of dominant mechanism for all three turbulent jets. For $m=0$ the map presents the shift in dominance of the $\mathrm{KH}$ at moderate Strouhal numbers to Orr at low frequencies, with mechanism shifts occurring at $S t \approx 0.3$ for $M=0.4,1.5$ and $S t \approx 0.45$ for $M=0.9$. For the lift-up mechanism, the region of dominance is rather consistent among all three jets and the region of lift-up dominance may be estimated as $S t_{L U} \leqslant 0.1 \mathrm{~m}$. It is important to stress that this map is an estimate of mechanism dominance and mechanisms are simultaneously present at the boundaries of dominant mechanism regions. As such, small changes in the metric values will lead to small changes in the cutoff regions for each mechanism, however, the general trends (i.e. Orr at low frequencies and the lift-up region scaling as $S t_{L U} \leqslant m$ ) reported are maintained.

\section{Local analysis for higher azimuthal modes}

Up to now, all results have used the full computational domain extending to 30 diameters downstream. The dominant azimuthal wavenumber for much of the frequency range is $m=1$, and as $S t \rightarrow 0$ the dominant azimuthal wavenumber is $m=2$. However, the question rises as to what the dominant azimuthal wavenumber is at various locations in the flow, particularly as $S t \rightarrow 0$.

To answer this question, we performed an SPOD analysis restricted to 2D crossstream planes throughout the range $x / D=[0.5-30]$. The three azimuthal wavenumbers exhibiting the largest energy are plotted versus $x / D$ in figure 12 . We see that the maximum energy for $x / D=30$ is inline with the global analysis result, occurring at an azimuthal wavenumber $m=2$; however, as the plane moves upstream we see a trend towards higher azimuthal wavenumbers. In fact, this trend scales as $m_{\max } \sim 1 / x+1$ 


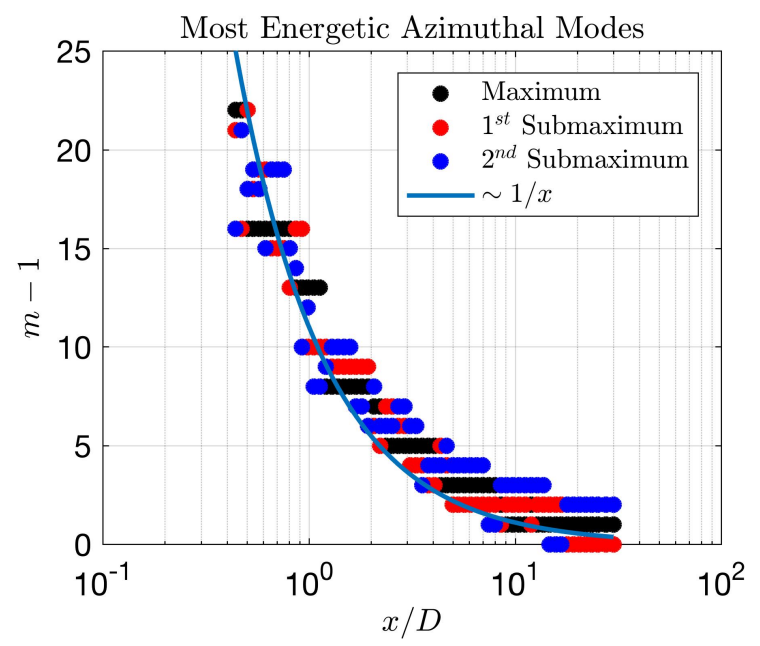

Figure 12: Wavenumbers of the three largest SPOD energies from the $S t \rightarrow 0$ SPOD bin as a function of streamwise distance. Here SPOD was performed locally on 2D streamwise cross sections and present a scaling of maximum azimuthal wavenumber as $\sim 1 / x+1$.

(i.e. the maximum wavenumber approaches 1). This scaling is inversely proportional to the linear scaling of the shear layer of the jet, $\sim x$ (Schmidt et al. 2018), and has also been reported from experimental observations of a turbulent Mach 0.4 jet by Nogueira et al. (2019) for axial stations $x / D \leqslant 8$. This suggests that the width of the shear layer determines support for particular azimuthal wavenumbers as $S t \rightarrow 0$.

Considering figure 12, one can observe that a local analysis allows for the identification of streaks of higher azimuthal wavenumbers. However, through the choice of a domain, which encompasses many diameters, the energetic effect of any given azimuthal wavenumber is integrated with all others. In our global analysis, we consider the Chu compressible energy norm. This norm greatly weights behavior found downstream that includes large spatial domains, which in turn contain significant amounts of energy. If one were interested in analyzing the role of azimuthal wavenumber $m=10$ streaks in turbulent jets, it is difficult to converge a coherent SPOD mode that is only energetic at $x / D$ around 1.25 through the use of a global domain.

To isolate streaks related to azimuthal wavenumbers $m=5,10$ the truncated domains $x=[0,2],[0,5],[0,10]$ are examined. Considering azimuthal wavenumbers $m=[0,15]$ we perform SPOD analyses on the truncated domains and first report the SPOD spectra and semi-discrete azimuthal wavenumber-frequency maps of relative energy (as a percentage) among wavenumbers at each frequency in figure 13 .

The SPOD spectra and wavenumber-frequency maps in figure 13 provide insight into both the total energy and the overall influence of each wavenumber as the domain is truncated towards the nozzle. The largest domain, $x / D=[0,10]$, shows energy peaking at low frequencies and dominated by low azimuthal wavenumbers, similar to the global spectrum in figure 3 . We also immediately see that the peak azimuthal wavenumber at $S t \rightarrow 0$ has shifted from $m=2$ to $m=4$, yet the high frequency behavior associated with $\mathrm{KH}$ remains active for low azimuthal wavenumbers, same as the $x / D=[0,30]$ analysis. Truncating the domain in half to $x / D=[0,5]$ presents a relative shift in total energy towards higher frequencies and the KH content maintains its strong $m=[0,1,2]$ behavior at these frequencies, while the distribution of energy at low frequencies is now centered 
a) SPOD Spectra
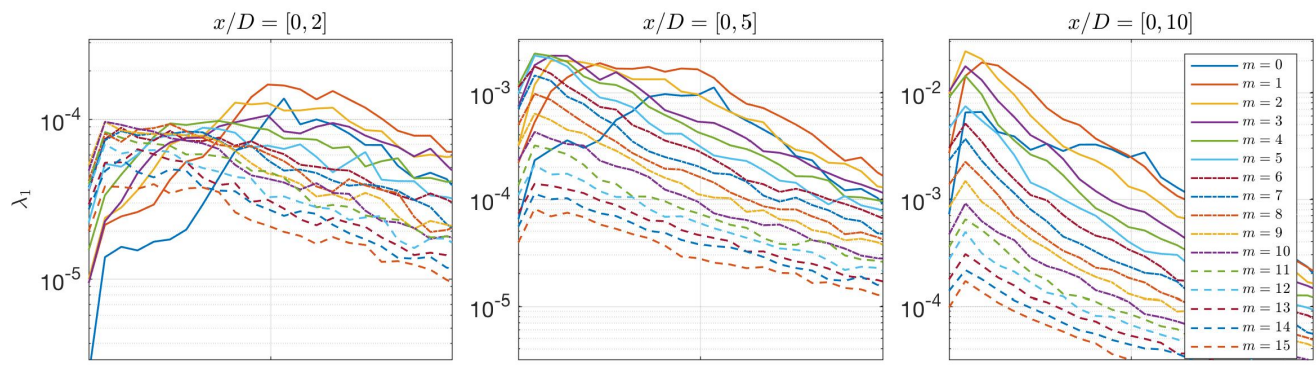

b) Wavenumber-frequency maps
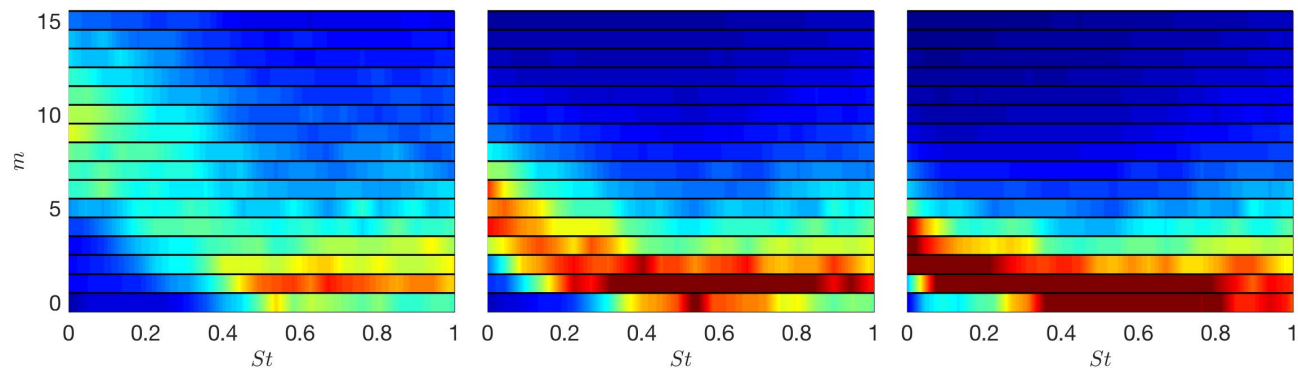

Figure 13: SPOD spectra of $\lambda_{1}$ for truncated domains of $x / D=[0,2],[0,5],[0,10]$ left to right respectively, for azimuthal wavenumbers $m=[0,15]$. a) SPOD spectrum curves for all wavenumbers. b) Semi-discrete wavenumber-frequency maps showing the distribution of energy (as a percentage) among wavenumbers at each frequency with contour levels at $0-20 \%$.

at $m=6$. The final truncation, $x / D=[0,2]$, shows a significant drop in energy at low frequencies and higher frequencies dominate the energy spectrum, however the peak maximum azimuthal wavenumber at low frequencies has shifted much higher, centered at $m=10$ near zero frequency. More importantly, the figures show a quite clear separation between the lift-up mechanism's influence in the low frequency portion of the energy map versus the KH mechanism's influence in higher frequency regions. This finding supports the idea that the large energies seen at moderately low frequency (i.e. $S t=[0, \approx 0.3]$ ) and non-zero azimuthal wavenumbers in the global sense are indeed a superposition of mechanisms in which the lift-up mechanism is a significant provider of energy, and perhaps, the largest contributor.

To determine the total energetic contribution of each azimuthal wavenumber in the three truncated domains, figure 14 presents the integrated energy of $\lambda_{1}$. Figure 14 a) gives the total energy of each azimuthal wavenumber with integration bounds of $S t=[0,1]$, where each domain has $m=1$ as the dominant wavenumber. This finding would usually be associated with the KH mechanism, but splitting the integration into $S t=[0,0.2]$ and $S t=[0.25,1]$ (figure $14 \mathrm{~b}$ ) and c) ) shows the contrary. The low frequency integration isolates energy due to the lift-up mechanism for each domain. This presents a rather smooth, Rayleigh-like distribution of energy among azimuthal wavenumbers with peaks greater than $m=1$, inline with the findings of Cavalieri et al. (2013) found at the lipline for PIV planes $x / D=[1,1.5,2,2.5,3]$. Additionally, the nonzero azimuthal energy peaks observed here also follow similar results of Citriniti \& George (2000) and Freund \& Colonius (2009), however, the exact azimuthal wavenumber peaks vary compared to these studies due to varying domains and norms used. The higher frequency integration then 
a)
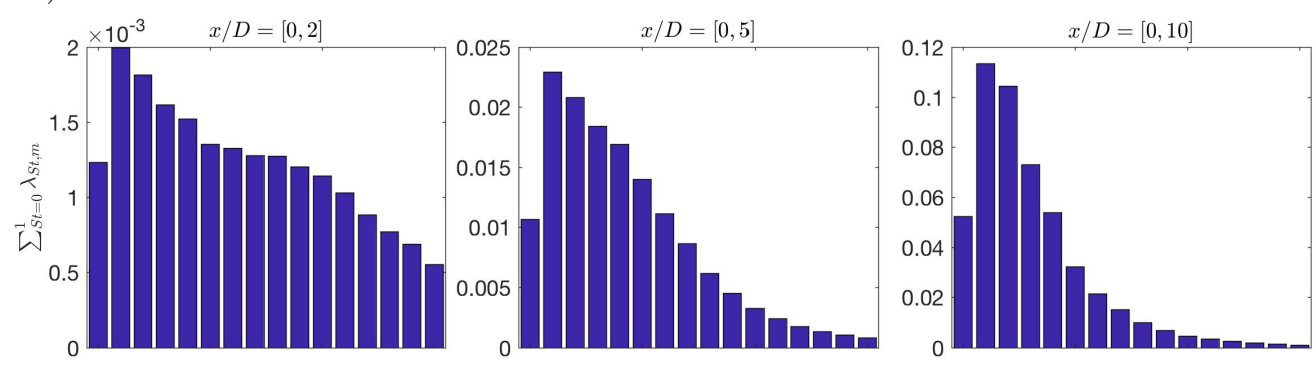

b)
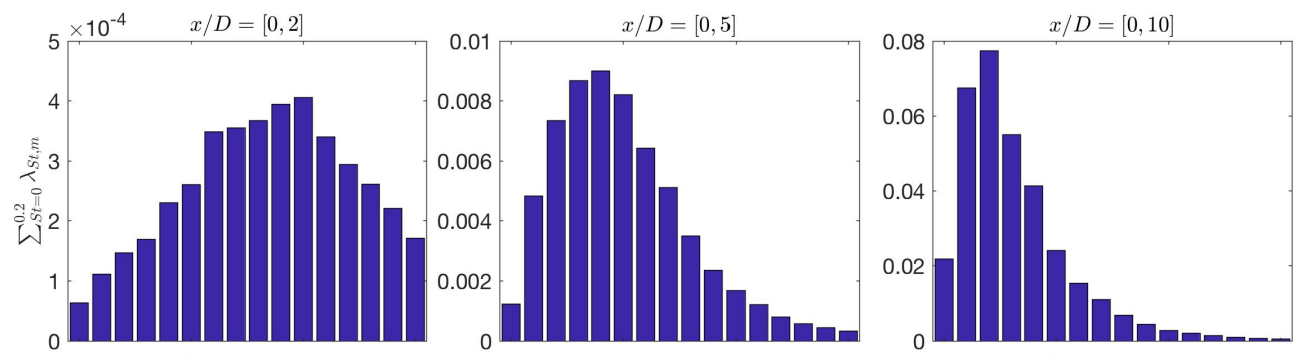

c)
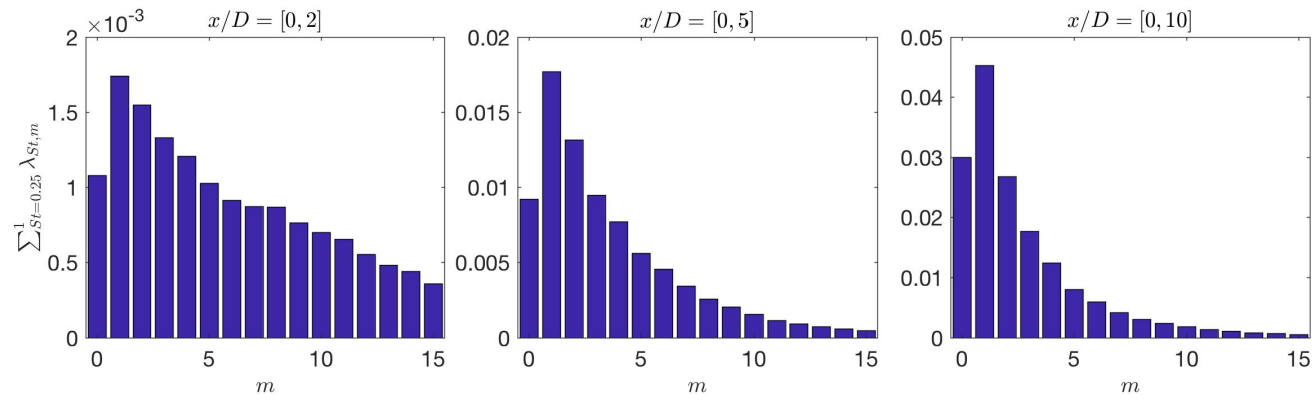

Figure 14: Integrated energy of $\lambda_{1}$ for each azimuthal wavenumber. a-c) present different bounds of integration in frequency with $S t=[0,1], S t=[0,0.2]$, and $S t=[0.25,1]$ respectively.

encompasses frequencies dominated with the $\mathrm{KH}$ mechanism, with all domains exhibiting the largest energy at $m=1$ and rapidly decaying for higher azimuthal wavenumbers. Considering we have already shown that lift-up is active at low frequencies and that the SPOD energy spectrum contains the largest energy contributions to the flow, the lift-up mechanism is now shown to be the greatest contributor to turbulent jet dynamics.

To further show that the $S t \rightarrow 0$ SPOD energies in this truncated domain analysis correspond to streaks, we show their 3D reconstructions of streamwise velocity and streamwise vorticity for $m=5,10$ in figure 15. It is worth noting that for these SPOD modes no special treatment for snapshot number or overlap was needed due to significant reductions in downstream energy that had previously hindered convergence. Therefore, SPOD parameters of 256 snapshots and a $50 \%$ overlap were employed.

All six plots shown for $m=5$ and 10 display smooth streaks of streamwise velocity, accompanied with streamwise rolls indicative of the lift-up mechanism, for all domains considered. Azimuthal wavenumber $m=5$ presents a case in which streaks are present with significant energy for each truncated domain, and as such, give rise to rather well 

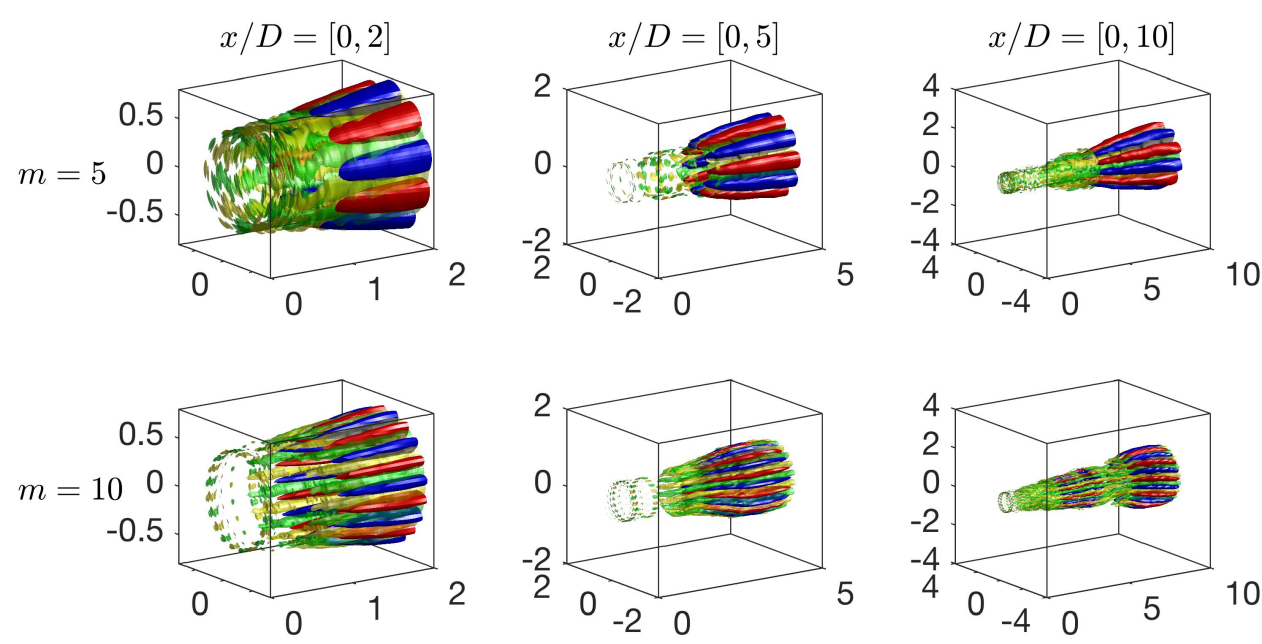

Figure 15: Three dimensional reconstruction of the first SPOD mode for $m=[5,10]$ (top and bottom, respectively) at three truncated domains $x / D=[0,2],[0,5],[0,10]$ (left to right, respectively) for $S t \rightarrow 0$. streamwise velocity, $u_{x}^{\prime}$, is denoted as red-blue with isosurfaces $\pm 25 \%$ the maximum streamwise velocity and streamwise vorticity, $\omega_{x}^{\prime}$, is shown as yellow-green with isosurfaces as $\pm 50 \%$ of the maximum streamwise vorticity.

defined streaks that inhabit the entire domain. For the $m=10$ streaks with truncated domains $x / D=[0,2]$ and $[0,5]$, both streamwise velocity streaks and streamwise vorticity rolls are easily identified. However, the $x / D=[0,10]$ domain gives a differing behavior. In the upstream region of the domain the streaks from the smaller domains are still readily present, however, another low-frequency, slowly-rotating streak enters towards the end of the domain. This can be attributed to the various domain and convergence issues described earlier, where a larger domain introduces additional energetic structures and noise which may be aliased into the $S t \rightarrow 0$ bin. Again, for the global results presented earlier in the paper, this was averted by increasing the number of snapshots in the DFT and reducing the bin sizes. Nevertheless, these results show that although high azimuthal wavenumbers do not appear to provide significant energy to the full flow field energy (e.g. figure 3), their energetic impact is significant in the near nozzle region and is a result of the lift-up mechanism.

\section{Conclusions \& Outlook}

We have extended the linear resolvent and data-driven SPOD analyses of turbulent jet mean flow fields to the zero-frequency limit. The main result is a confirmation and extension of the local analysis of Nogueira et al. (2019), namely the identification of the lift-up mechanism as an important amplifier of disturbances in turbulent jets. We found lift-up responsible for the generation of streamwise elongated structures, known as streaks, at low frequency, nonzero azimuthal wavenumbers for turbulent round jets at Mach numbers $0.4,0.9$, and 1.5. At higher frequencies, $\mathrm{KH}$ becomes the globally dominant mechanism, and the Orr mechanism is active over all frequencies but plays a subdominant role at those frequencies and azimuthal wavenumbers where lift-up and $\mathrm{KH}$ are active. For all regimes, there is a reasonable agreement between the resolvent analysis and SPOD modes from the associated LES database, confirming that the theoretical mechanisms 
are active (and dominant) in the turbulent regime. This agreement is predicated on the addition of an eddy viscosity model in the linear resolvent Morra et al. (2019); Pickering et al. (2019). While the simple model we employed suffices to establish the link between theory and observation, further refinements to the model would be required to establish a resolvent analysis that is predictive of turbulence structure.

Streaks observed using SPOD show similar structure to (space-only) POD modes reported by Freund \& Colonius (2009) and SPOD modes with limited spatial extent by Citriniti \& George (2000), and predicted by resolvent analyses for $m>0$ at $S t=0$. Both SPOD and resolvent modes provide significant qualitative agreement in both streamwise vorticity and streamwise velocity, which are related to rolls and streaks, respectively. The resolvent results show optimal forcing in the form of streamwise vortices ( rolls, $f_{\omega_{x}}$ ) from the nozzle exit decaying slowly downstream, followed by a response of streamwise vortices $\left(q_{\omega_{x}}\right)$, and finally, further downstream appears a response of streamwise velocity $\left(q_{u_{x}}\right)$, or streaks. These characterizations of the flow, from both resolvent and SPOD, now link multiple previous experimental and numerical observations in transitional and turbulent jets of streamwise vortices (Liepmann 1991; Martin \& Meiburg 1991; Paschereit et al. 1992; Liepmann \& Gharib 1992; Arnette et al. 1993) and streaks (Citriniti \& George 2000; Caraballo et al. 2003; Freund \& Colonius 2009; Cavalieri et al. 2013) to the lift-up mechanism.

An analysis of mode shapes, spatial energy content, and sensitivity maps allowed us to characterize the portions of wavenumber-frequency space where the different mechanisms are dominant. The behavior of the $m=0$ response is unique, as axisymmetric streaks cannot exist; rather the Orr response is dominant for low and high frequencies, with the $\mathrm{KH}$ response dominating over an intermediate frequency regime centered on $S t=0.6$. For non-axisymmetric modes, the lift-up mechanism, and resulting streak response, is dominant at low frequencies, although with progressively higher wavenumber, these are limited in spatial extent to nearer the nozzle exit. We find that the azimuthal wavenumber of dominant streak is inversely proportional to shear layer widthand scales with $\sim 1 / x$. For the lower azimuthal wavenumbers, the lift-up response is overall most energetic structure in the global jet (over all frequencies).

The presence of the lift-up mechanism in turbulent jets suggests further investigation of the resulting dynamics, and its potential impact for control of quantities such as jet noise. Considering streaks are highly energetic structures it is likely that their behavior (i.e. breakdown and regeneration) have significant impact on other structures and phenomenon in turbulent jets, such as Orr and $\mathrm{KH}$ wavepackets and qualities associated with these structures' intermittency. In fact, noise reduction has been accomplished via the introduction of streamwise vortices at the nozzle exit using tabs (Samimy et al. 1993; Zaman et al. 1994; Zaman 1999) and chevrons (Saiyed et al. 2003; Callender et al. 2005; Alkislar et al. 2007). Recently, Rigas et al. (2019) showed the presence of both chevron induced streamwise vortices and their accompanying streaks in the baseflow of a turbulent, chevron jet, while Marant \& Cossu (2018) have reported the ability of streaks to stabilize the KH instability for a planar laminar shear flow. Considering past computational work, using PSE, by Sinha et al. (2016) demonstrated the ability of chevrons to stabilize $\mathrm{KH}$ wavepackets, the dominant mechanism in jet noise Jordan \& Colonius (2013), it is now likely that lift-up and streaks are the mechanism behind such jet noise reduction techniques. 

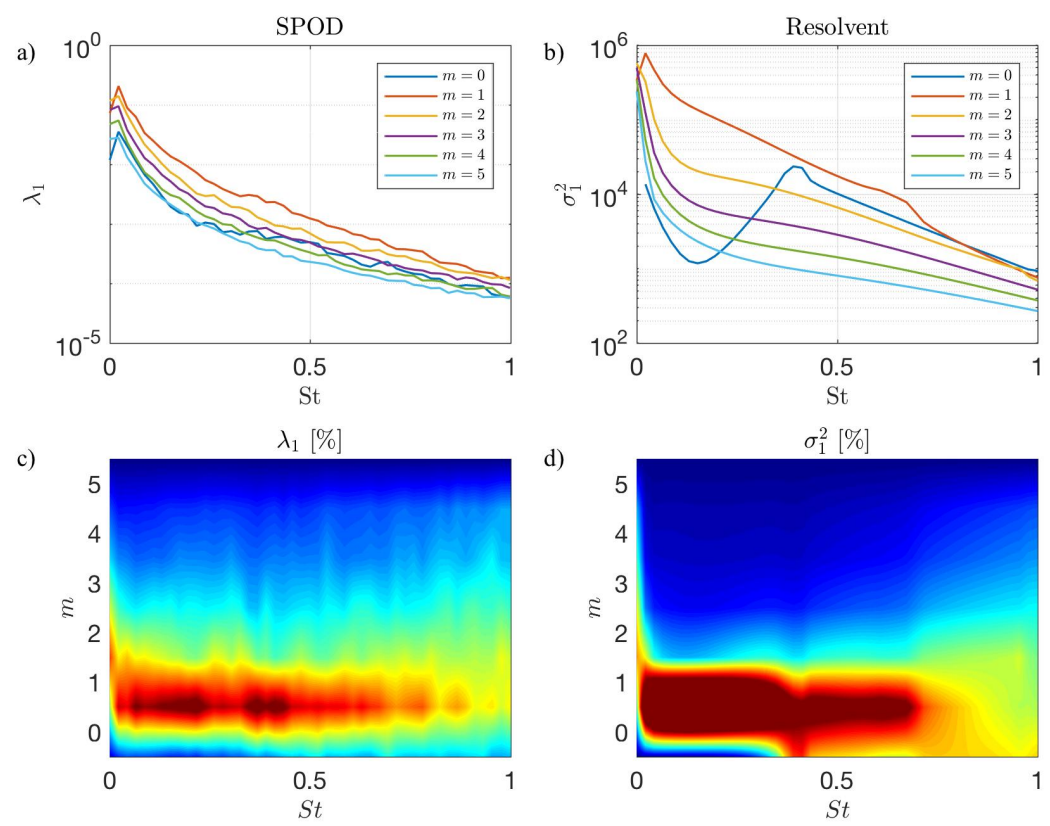

Figure 16: Modal energy from SPOD and resolvent analyses of a Mach 0.9 round jet.

\section{Acknowledgments}

This research was supported by a grant from the Office of Naval Research (grant No. N00014-16-1-2445) with Dr. Steven Martens as program manager. E.P. was supported by the Department of Defense (DoD) through the National Defense Science \& Engineering Graduate Fellowship (NDSEG) Program. The LES study was performed at Cascade Technologies, with support from ONR and NAVAIR SBIR project, under the supervision of Dr. John T. Spyropoulos. The main LES calculations were carried out on DoD HPC systems in ERDC DSRC.

\section{Appendix A. Transonic and supersonic jets}

This section presents similar results supporting the presence of the lift-up mechanisms and streaks for Mach 0.9 and Mach 1.5 turbulent round jets. We first show the azimuthal wavenumber-frequency maps for each of the jets in figure 16 and figure 17. For both figures, there is significant qualitative agreement between the SPOD energies and the resolvent gains.

In the Mach 0.9 jet we see a small spike at $S t \sim 0.4$ for the resolvent analysis due to trapped acoustic modes, that is slightly over predicted compared to SPOD. Another small discrepancy between SPOD and resolvent is the additional influence of the $m=0$ mode in the resolvent analysis when compared to SPOD. However, both show large energies at low frequencies and display behavior similar to the Mach 0.4 jet with energy peaking at $m=2$ as $S t \rightarrow 0$.

For the Mach 1.5 jet there is a similar over-prediction of the resolvent $m=0$ case when compared to SPOD. Despite this, the remainder of the resolvent map follows SPOD characteristics quite well. For high frequencies, energy is rather equally distributed among 

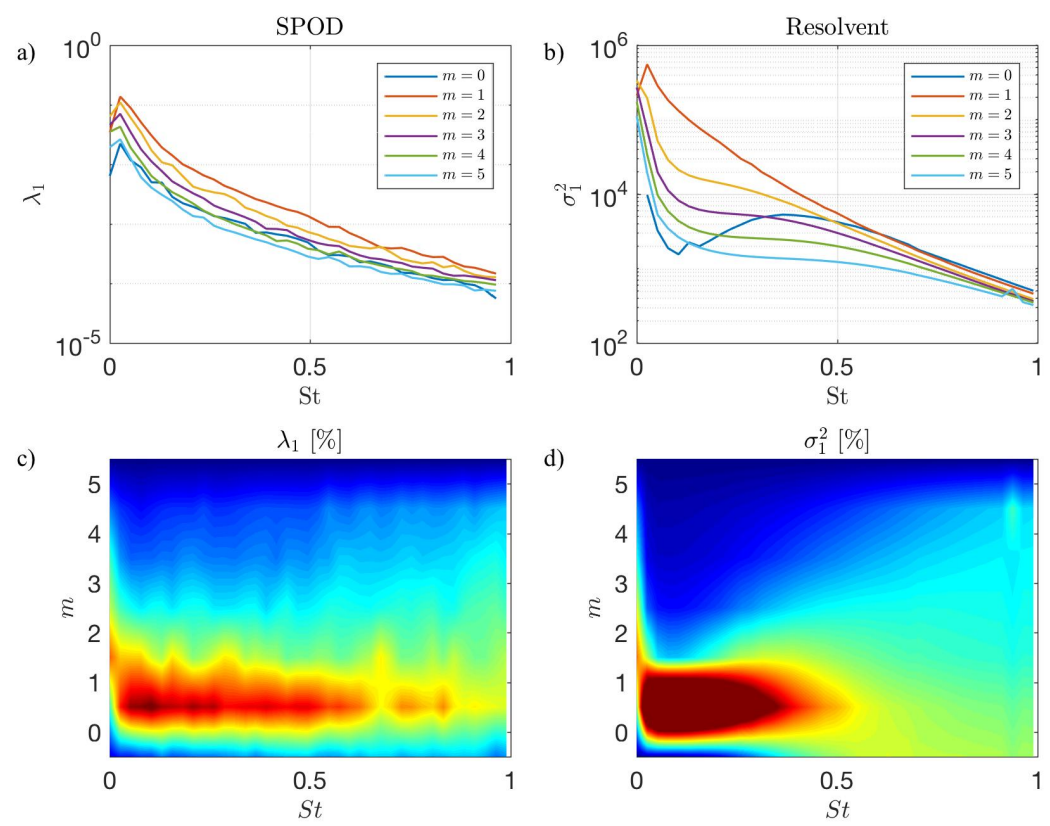

Figure 17: Modal energy from SPOD and resolvent analyses of a Mach 1.5 round jet.

the azimuthal wavenumbers and at low frequencies both analyses display high energy behavior similar to the Mach 0.4 jet with energy peaking at $m=2$ as $S t \rightarrow 0$.

We show the 3D reconstructions of the SPOD modes for $m=1,3$ shown in figure 18 using 1024 snapshots and $75 \%$ overlap. All four plots show streaks of streamwise velocity response along with the associated streamwise vorticity, with the clearest descriptions shown for the $m=3$ cases. The $m=3$ wavenumber plots for both jets present streaky structures paired with streamwise vorticity rolls placed perfectly between each streak. The $m=1$ plots are not quite as appealing, but both plots present streaks in streamwise velocity and are also paired with streamwise vorticity placed between each streak.

Finally, we show the resolvent analysis at $S t=0$ for both jets and wavenumbers $m=1,3$ in figure 19. Here we show the same behavior as previously described for the Mach 0.4 jet. Forcing in the form of streamwise vorticity begins upstream near the nozzle, creates responses of streamwise rolls, which lead to lift-up and large streamwise velocity responses. Considering all of the above evidence in Mach 0.9 and 1.5 jets, we conclude that the lift-up mechanism is present in all turbulent jets.

\section{Appendix B. SPOD and resolvent semi-discrete energy maps for Mach 0.4}

Figure 20 gives the semi-discrete, continuous in frequency and discrete in azimuthal wavenumber, representation of figure 3 .

Abreu, L. I., Cavalieri, A. V. G., Schlatter, P., Vinuesa, R. \& Henningson, D. 


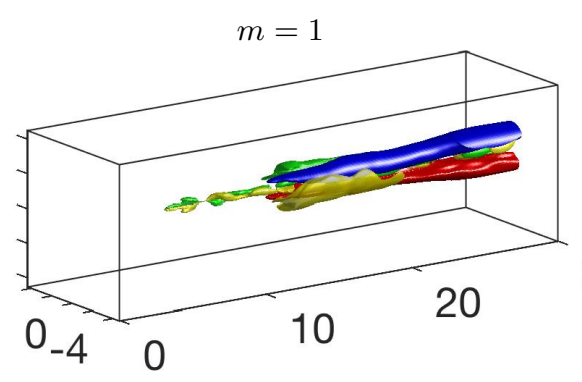

a) Mach $0.9 \quad m=3$

$m=1$

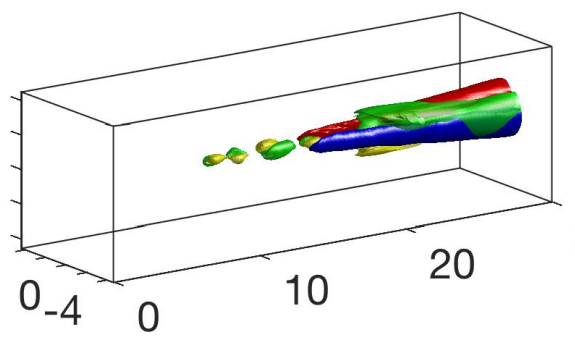

30

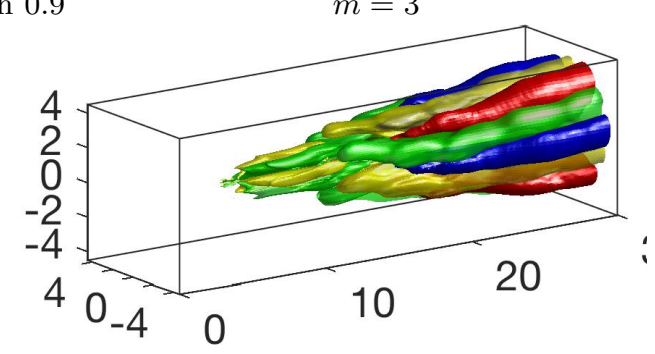

b) Mach 1.5

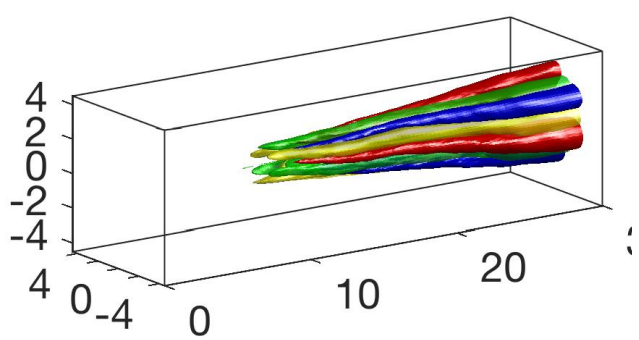

Figure 18: Three dimensional reconstruction of the first SPOD mode (streamwise velocity, $u_{x}^{\prime}$, red-blue, streamwise vorticity, $\omega_{x}^{\prime}$, yellow-green) as $S t \rightarrow 0$ for $m=1$ (left column) and $m=3$ (right column) using isosurfaces of $\pm 50 \%$ of the maximum streamwise velocity and isosurfaces of $\pm 25 \%$ of the maximum streamwise vorticity, with the exception of the Mach $1.5, m=1$, case where red-blue isosurfaces are instead $\pm 30 \%$ of the maximum streamwise velocity.

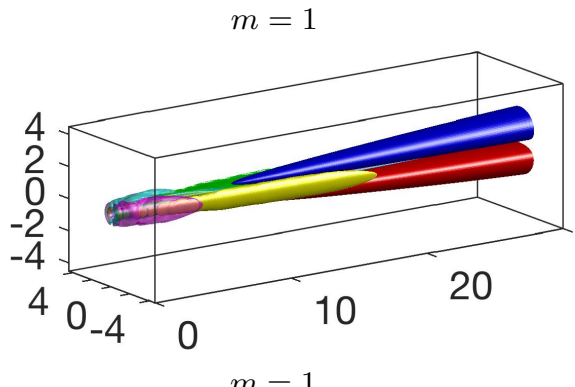

a) Mach 0.9

$m=3$

30
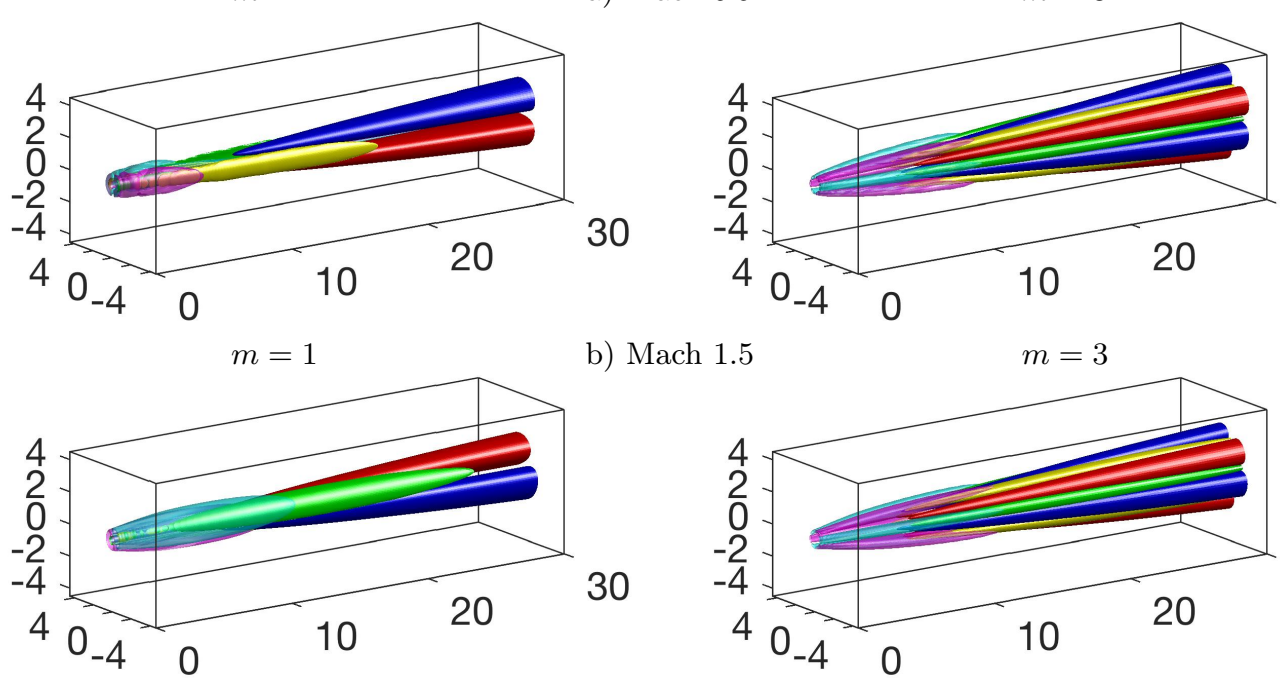

b) Mach 1.5

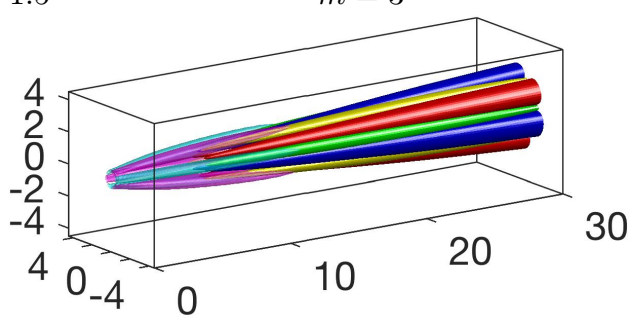

Figure 19: Global resolvent forcing and response for $m=1$ (left) and $m=3$, (right) at $S t=0$. The streamwise forcing vorticity is shown in magenta-cyan with isosurfaces $\pm 0.05\left\|f_{\omega_{x}}\right\|_{\infty}$ for $m=1$ and $\pm 0.2\left\|f_{\omega_{x}}\right\|_{\infty}$ for $m=3$, streamwise response vorticity is shown in yellow-green with isosurfaces $\pm 0.5\left\|q_{\omega_{x}}\right\|_{\infty}$, and streamwise response velocity is shown in red-blue with isosurfaces $\pm 0.25\left\|q_{u_{x}}\right\|_{\infty}$. 

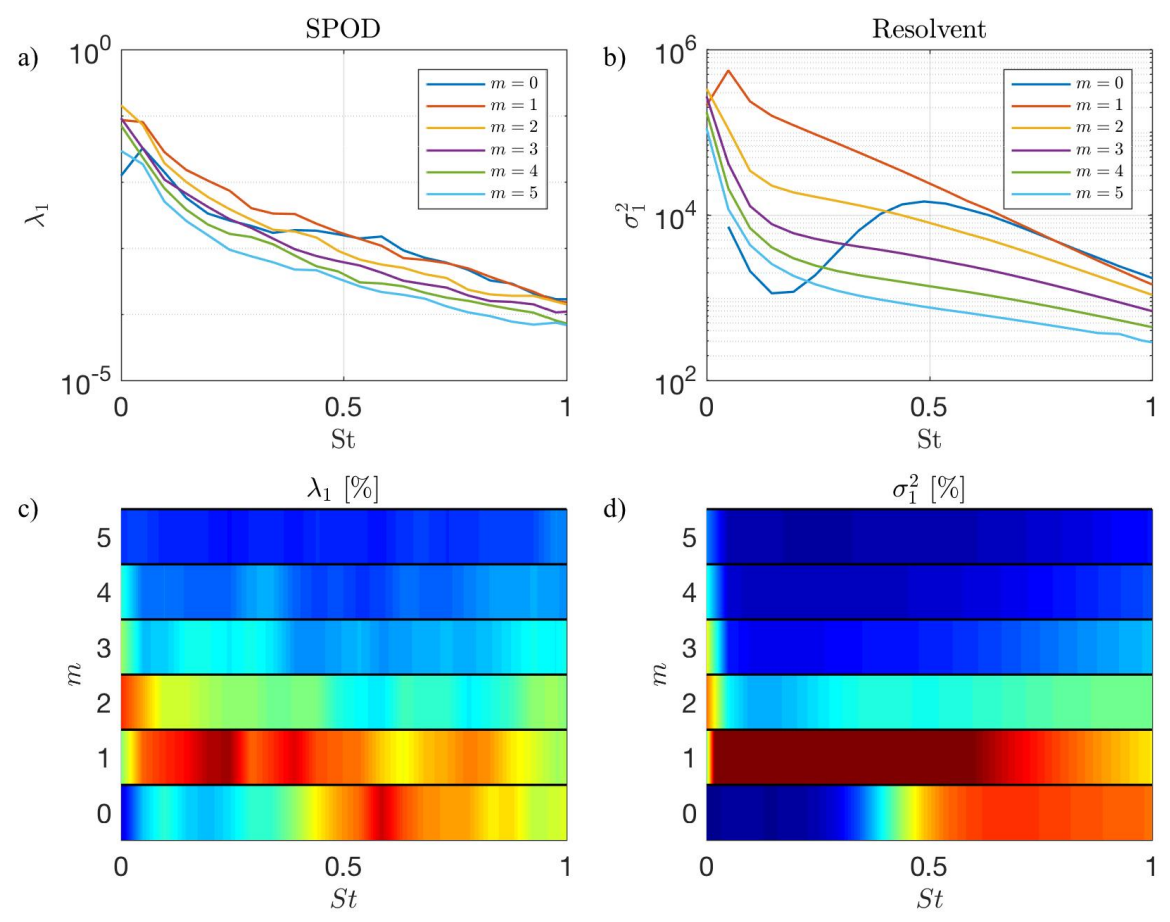

Figure 20: Modal energy from SPOD and resolvent analyses of a Mach 0.4 round jet, shown here in semi-discrete form.

2019 Reduced-order models to analyse coherent structures in turbulent pipe flow. In 11th International Symposium on Turbulence and Shear Flow Phenomena.

Alkislar, M. B., Krothapalli, A. \& Butler, G. W. 2007 The effect of streamwise vortices on the aeroacoustics of a mach 0.9 jet. Journal of Fluid Mechanics 578, 139-169.

Arnette, S. A., Samimy, M. \& Elliott, G. S. 1993 On streamwise vortices in high reynolds number supersonic axisymmetric jets. Physics of Fluids A: Fluid Dynamics 5 (1), 187-202.

BRANDT, L. 2014 The lift-up effect: the linear mechanism behind transition and turbulence in shear flows. European Journal of Mechanics-B/Fluids 47, 80-96.

Brès, G. A., Bose, S. T., Emory, M., Ham, F. E., Schmidt, O. T., Rigas, G. \& Colonius, T. 2018 Large eddy simulations of co-annular turbulent jet using a voronoibased mesh generation framework. AIAA paper 2018-3302.

Brès, G. A., Ham, F. E., Nichols, J. W. \& Lele, S. K. 2017 Unstructured large-eddy simulations of supersonic jets. AIAA Journal pp. 1164-1184.

Brès, G. A., Jordan, P., Jaunet, V., Le Rallic, M., Cavalieri, A. V. G., Towne, A., Lele, S. K., Colonius, T. \& Schmidt, O. T. 2018 Importance of the nozzle-exit boundary-layer state in subsonic turbulent jets. J. Fluid Mech. 851, 83-124.

Brown, G. L. \& Roshko, A. 1974 On density effects and large structure in turbulent mixing layers. Journal of Fluid Mechanics 64 (4), 775-816.

Butler, K. M. \& Farrell, B. F. 1992 Three-dimensional optimal perturbations in viscous shear flow. Physics of Fluids A: Fluid Dynamics 4 (8), 1637-1650.

Callender, B., Gutmark, E. J. \& Martens, S. 2005 Far-field acoustic investigation into chevron nozzle mechanisms and trends. AIAA journal 43 (1), 87-95.

Caraballo, E., Samimy, M., Scott, J., Narayanan, S. \& DeBonis, J. 2003 Application of 
proper orthogonal decomposition to a supersonic axisymmetric jet. AIAA journal 41 (5), 866-877.

Cavalieri, A. V. G., Rodríguez, D., Jordan, P., Colonius, T. \& Gervais, Y. 2013 Wavepackets in the velocity field of turbulent jets. J. Fluid Mech. 730, 559-592.

Chu, B.-T. 1965 On the energy transfer to small disturbances in fluid flow (part i). Acta Mechanica 1 (3), 215-234.

Citriniti, J. H. \& George, W. K. 2000 Reconstruction of the global velocity field in the axisymmetric mixing layer utilizing the proper orthogonal decomposition. J. Fluid Mech. 418, 137-166.

Crighton, D. G. \& Gaster, M. 1976 Stability of slowly diverging jet flow. Journal of Fluid Mechanics $\mathbf{7 7}$ (2), 397-413.

Crow, S. C. \& Champagne, F. H. 1971 Orderly structure in jet turbulence. Journal of Fluid Mechanics 48 (3), 547-591.

Eitel-Amor, G., Örlü, R. \& Schlatter, P. 2014 Simulation and validation of a spatially evolving turbulent boundary layer up to $\operatorname{Re} \theta=8300$. International Journal of Heat and Fluid Flow 47, 57-69.

Ellingsen, T. \& Palm, E. 1975 Stability of linear flow. Phys. Fluids 18 (4), 487-488.

Farrell, B. F. \& IoAnnou, P. J. 1993 Optimal excitation of three-dimensional perturbations in viscous constant shear flow. Physics of Fluids A: Fluid Dynamics 5 (6), 1390-1400.

Freund, J. B. \& Colonius, T. 2009 Turbulence and sound-field pod analysis of a turbulent jet. Int. J. Aeroacoust. 8 (4), 337-354.

Garnaud, X., Lesshafft, L., Schmid, P. J. \& Huerre, P. 2013 Modal and transient dynamics of jet flows. Phys. Fluids 25 (4), 044103.

Gudmundsson, K. \& Colonius, T. 2011 Instability wave models for the near-field fluctuations of turbulent jets. Journal of Fluid Mechanics 689, 97-128.

Hellström, L. H. O., Sinha, A. \& Smits, A. J. 2011 Visualizing the very-large-scale motions in turbulent pipe flow. Phys. Fluids 23 (1), 011703.

Hutchins, N. \& Marusic, I. 2007 Evidence of very long meandering features in the logarithmic region of turbulent boundary layers. J. Fluid Mech. 579, 1-28.

Hwang, Y. \& Cossu, C. 2010 Amplification of coherent streaks in the turbulent couette flow: an input-output analysis at low reynolds number. Journal of Fluid Mechanics 643, 333348.

Jaunet, V., Jordan, P. \& Cavalieri, A. V. G. 2017 Two-point coherence of wave packets in turbulent jets. Physical Review Fluids 2 (2), 024604.

Jeun, J., Nichols, J. W. \& Jovanović, M. R. 2016 Input-output analysis of high-speed axisymmetric isothermal jet noise. Phys. Fluids 28 (4), 047101.

Jiménez, J. 2018 Coherent structures in wall-bounded turbulence. Journal of Fluid Mechanics 842.

Jordan, P. \& Colonius, T. 2013 Wave packets and turbulent jet noise. Annu. Rev. Fluid Mech. 45, 173-195.

Kim, H. T., Kline, S. J. \& Reynolds, W. C. 1971 The production of turbulence near a smooth wall in a turbulent boundary layer. Journal of Fluid Mechanics 50 (1), 133-160.

Klebanoff, P. S. 1971 Effect of freestream turbulence on the laminar boundary layer. Bulletin of the American Physical Society 16, 1321.

LANDAhL, M. T. 1980 A note on an algebraic instability of inviscid parallel shear flows. J. Fluid Mech. 98 (2), 243-251.

Lesshafft, L., Semeraro, O., Jaunet, V., Cavalieri, A. V. G. \& Jordan, P. 2019 Resolvent-based modelling of coherent wavepackets in a turbulent jet. Phys. Rev. Fluids $4(6), 063901$.

Liepmann, D. 1991 Streamwise vorticity and entrainment in the near field of a round jet. Physics of Fluids A: Fluid Dynamics 3 (5), 1179-1185.

Liepmann, D. \& Gharib, M. 1992 The role of streamwise vorticity in the near-field entrainment of round jets. Journal of Fluid Mechanics 245, 643-668.

Lumley, J. L. 1967 The structure of inhomogeneous turbulent flows. Atmospheric turbulence and radio propagation pp. $166-178$.

Lumley, J. L. 1970 Stochastic tools in turbulence. New York: Academic Press. 
Marant, M. \& Cossu, C. 2018 Influence of optimally amplified streamwise streaks on the kelvin-helmholtz instability. J. Fluid Mech. 838, 478-500.

Martin, J. E. \& Meiburg, E. 1991 Numerical investigation of three-dimensionally evolving jets subject to axisymmetric and azimuthal perturbations. Journal of Fluid Mechanics 230, 271-318.

McKeon, B. J. \& Sharma, A. S. 2010 A critical-layer framework for turbulent pipe flow. J. Fluid Mech. 658, 336-382.

Moffatt, H. K. 1965 The interaction of turbulence with strong wind shear. Atmospheric Turbulence and Radio Waves Propagation, Proc. Intern. Collq. Moscow, 1965 pp. 139156.

Mollo-Christensen, E. 1967 Jet noise and shear flow instability seen from an experimenter's viewpoint .

Monokrousos, A., Åkervik, E., Brandt, L. \& Henningson, D. S. 2010 Global threedimensional optimal disturbances in the blasius boundary-layer flow using time-steppers. J. Fluid Mech. 650, 181-214.

Monty, J. P., Stewart, J. A., Williams, R. C. \& Chong, M. S. 2007 Large-scale features in turbulent pipe and channel flows. J. Fluid Mech. 589, 147-156.

Morra, P., Semeraro, O., Henningson, D. S. \& Cossu, C. 2019 On the relevance of reynolds stresses in resolvent analyses of turbulent wall-bounded flows. J. Fluid Mech. 867, 969-984.

Nogueira, P. A. S., Cavalieri, A. V. G., Jordan, P. \& Jaunet, V. 2019 Large-scale, streaky structures in turbulent jets. Journal of Fluid Mechanics 873, 211-237.

Paschereit, C. O., Oster, D., Long, T. A., Fiedler, H. E. \& Wygnanski, I. 1992 Flow visualization of interactions among large coherent structures in an axisymmetric jet. Experiments in Fluids 12 (3), 189-199.

Pickering, E., Rigas, G., Sipp, D., Schmidt, O. T. \& Colonius, T. 2019 Eddy viscosity for resolvent-based jet noise models. In 25th AIAA/CEAS Aeroacoustics Conference, p. 2454.

QADRI, U. A. \& Schmid, P. J. 2017 Frequency selection mechanisms in the flow of a laminar boundary layer over a shallow cavity. Phys. Rev. Fluids 2, 013902.

Rigas, G., Pickering, E., Schmidt, O. T., Nogueira, P. A., Cavalieri, A. V., Brès, G. A. \& Colonius, T. 2019 Streaks and coherent structures in jets from round and serrated nozzles. In 25th AIAA/CEAS Aeroacoustics Conference, p. 2597.

Saiyed, N. H., Mikkelsen, K. L. \& Bridges, J. E. 2003 Acoustics and thrust of quiet separate-flow high-bypass-ratio nozzles. AIAA journal 41 (3), 372-378.

Samimy, M., Zaman, K. \& Reeder, M.F. 1993 Effect of tabs on the flow and noise field of an axisymmetric jet. AIAA journal 31 (4), 609-619.

Schmidt, O. T., Towne, A., Rigas, G., Colonius, T. \& Brès, G. A. 2018 Spectral analysis of jet turbulence. J. Fluid Mech. 855, 953-982.

Semeraro, O., Jaunet, V., Jordan, P., Cavalieri, A. V. \& Lesshafft, L. 2016 Stochastic and harmonic optimal forcing in subsonic jets. In 22nd AIAA/CEAS Aeroacoustics Conference, p. 2935.

Sinha, A., Rajagopalan, A. \& Singla, S. 2016 Linear stability implications of chevron geometry modifications for turbulent jets. In 22nd AIAA/CEAS Aeroacoustics Conference, p. 3053.

Tissot, G., Lajús JR, F. C., Cavalieri, A. V. G. \& Jordan, P. 2017 Wave packets and orr mechanism in turbulent jets. Phys. Rev. Fluids 2 (9), 093901.

Towne, A., Schmidt, O. T. \& Colonius, T. 2018 Spectral proper orthogonal decomposition and its relationship to dynamic mode decomposition and resolvent analysis. J. Fluid Mech. $\mathbf{8 4 7}, 821-867$.

ZAMAN, K. 1999 Spreading characteristics of compressible jets from nozzles of various geometries. Journal of Fluid mechanics 383, 197-228.

Zaman, K., Reeder, M. F. \& Samimy, M. 1994 Control of an axisymmetric jet using vortex generators. Physics of Fluids 6 (2), 778-793. 